\title{
Article
}

\section{Manipulation-free cultures of human iPSC-derived cardiomyocytes offer a novel screening method for cardiotoxicity}

\author{
Sheeja RAJASINGH ${ }^{1, \#}$, Dona Greta ISAl ${ }^{2, \#}$, Saheli SAMANTA ${ }^{1}$, Zhi-gang ZHOU ${ }^{1}$, Buddhadeb DAWN ${ }^{1}$, William H KINSEY², \\ Andras CZIROK ${ }^{2, *}$, Johnson RAJASINGH ${ }^{1,3, *}$ \\ ${ }^{1}$ Cardiovascular Research Institute, Division of Cardiovascular Diseases, Department of Internal Medicine; ${ }^{2}$ Department of Anatomy \\ and Cell Biology; ${ }^{3}$ Department of Biochemistry and Molecular Biology, University of Kansas Medical Center, Kansas City, KS, USA
}

\begin{abstract}
Induced pluripotent stem cell (iPSC)-based cardiac regenerative medicine requires the efficient generation, structural soundness and proper functioning of mature cardiomyocytes, derived from the patient's somatic cells. The most important functional property of cardiomyocytes is the ability to contract. Currently available methods routinely used to test and quantify cardiomyocyte function involve techniques that are labor-intensive, invasive, require sophisticated instruments or can adversely affect cell vitality. We recently developed optical flow imaging method analyses and quantified cardiomyocyte contractile kinetics from video microscopic recordings without compromising cell quality. Specifically, our automated particle image velocimetry (PIV) analysis of phase-contrast video images captured at a high frame rate yields statistical measures characterizing the beating frequency, amplitude, average waveform and beatto-beat variations. Thus, it can be a powerful assessment tool to monitor cardiomyocyte quality and maturity. Here we demonstrate the ability of our analysis to characterize the chronotropic responses of human iPSC-derived cardiomyocytes to a panel of ion channel modulators and also to doxorubicin, a chemotherapy agent with known cardiotoxic side effects. We conclude that the PIV-derived beat patterns can identify the elongation or shortening of specific phases in the contractility cycle, and the obtained chronotropic responses are in accord with known clinical outcomes. Hence, this system can serve as a powerful tool to screen the new and currently available pharmacological compounds for cardiotoxic effects.
\end{abstract}

Keywords: iPSCs; stem cells; cardiomyocytes; contractility; optical flow imaging method; cardiotoxicity; ion channel modulator; doxorubicin

Acta Pharmacologica Sinica (2018) 39: 1590-1603; doi: 10.1038/aps.2017.183; published online 5 Apr 2018

\section{Introduction}

Cardiovascular toxicity, a serious complication encountered during drug therapy, can lead to cardiomyopathy and heart failure. Many previously approved and marketed cardiac drugs have been withdrawn from the market due to severe side effects that became apparent only after the drugs were used in the clinics ${ }^{[1]}$. Developing a new drug is a very expensive and lengthy process-the current estimated cost to accomplish the Food \& Drug Administration approval is about 1.5 billion $\mathrm{USD}^{[2]}$. Thus, substantial resources are wasted due to the limited predictive power of preclinical cardiotoxicity

\footnotetext{
\# These authors contributed equally to this work.

* To whom correspondence should be addressed.

E-mail rjohnson9@kumc.edu (Johnson RAJASINGH); aczirok@gmail.com (Andras CZIROK)

Received 2017-08-01 Accepted 2017-12-31
}

screening techniques. Therefore, it has been a persistent challenge for the pharmaceutical industry to either develop safe drugs that have no cardiac toxicity, or to optimize treatment combinations utilizing multiple agents that minimize druginduced damage to the heart. The most common harmful side effects which involve cardiac arrhythmias ${ }^{[3]}$, thus, there is a sustained need for preclinical (in vitro) drug screening assays that are especially sensitive to identify chronotropic effects and drug toxicity at the early stage of the drug development process.

Currently, the routinely used cardiotoxicity screening techniques involve sophisticated instruments and require some manipulation of the cells: clamping with electrodes, using dyes for intracellular imaging or mechanical indentation ${ }^{[4]}$. These methods are labor-intensive, invasive and can affect the cells' viability. Our recently developed method to quantify cardiomyocyte (CMC) contraction from video microscopic 
recording is manipulation free and technically simple. Thus, it is a promising approach to monitor CMC quality and maturity without compromising cell quality ${ }^{[5]}$.

Primary human CMCs are difficult to obtain and cannot be kept for prolonged time periods in culture, hence they are not optimal for preclinical cardiotoxicity screening. Human induced pluripotent stem cell (iPSC)-derived cardiomyocytes (iCMCs), in contrast, could provide an unlimited cell source for regenerative medicine and cardiotoxicity screens ${ }^{[6-8]}$. Earlier reports demonstrated that several drugs affect $\mathrm{CMC}$ beating frequency irrespective whether they were derived from embryonic or induced pluripotent stem cells, and are also compatible with clinical observations ${ }^{[9-11]}$.

Most studies on iCMC responses, however, were utilizing electrophysiological readouts and the actual contractile kinetics of CMCs was ignored. We propose that our previously established optical-flow based technique of measuring iCMCs contractility kinetics can be a beneficial tool for preclinical drug screening. The method extracts a functional phenotype of the CMC culture and does not require manipulation of the cells. To determine the utility of our approach, we have evaluated how iCMC cultures respond to various pharmacological modulators of calcium (isoproterenol), potassium (NS1643, E4031) and sodium (veratridine, mexiletine) transport. We demonstrate that our assay successfully reproduced previously reported chronotropic effects. We trust that the hereby demonstrated reliability will motivate the application of the method to the early detection and prevention of cardiotoxic risk.

\section{Materials and methods}

\section{Data availability}

The source code of our analysis software is posted at https:/ / github.com/aczirok/piv-div, imaging data is shared at https://osf.io/qvdhn.

\section{Antibodies and reagents}

We used primary antibodies for cardiotroponin T (CTT), alpha sarcomeric actin (a-SA), Gata4 (Santa Cruz Biotechnology, Inc), anti-nNav1.5 antibody (4G8:1G7), anti-Kv1.1 potassium channel antibody and anti-CACNA1S antibody 1A (Abcam) to perform in vitro analysis. As secondary antibodies, we used HRP-conjugated donkey anti-mouse, anti-rabbit, anti-goat (Santa Cruz Biotechnology, Inc); TRITC-, FITC-, and Cy-5-conjugated donkey anti-mouse, anti-goat, and anti-rabbit (Jackson ImmunoResearch Laboratories, Inc) antibodies. We also used DAPI (Life-Tech); Matrigel (BD Biosciences) Nutristem medium (Stemgent, Cambridge, MA, USA) and FACS buffer (BD Biosciences).

\section{Chronotropic compounds}

Several compounds were used to elicit changes in the spontaneous beating activity of iCMC cultures. The concentration for each drug was selected based on the review of cardio-active effects in previous studies ${ }^{[12-14]}$. Isoproterenol (Abcam) is a selective $\beta$-agonist class IV anti-arrhythmic calcium channel activator which is used for the treatment of mild or transient episodes of heart block and bradycardia. NS1643 (Abcam) is an anti-arrhythmic activator of hERG $\mathrm{K}^{+}$channels. E4031 (Abcam) is a methanesulfonanilide class III anti-arrhythmic drug that blocks hERG-type potassium channels. Veratridine (Abcam) is an activator for voltage gated $\mathrm{Na}^{+}$channels. Mexiletine (Abcam) is a voltage gated $\mathrm{Na}^{+}$channel blocker which belongs to the class IB anti-arrhythmic group of medicines.

\section{Differentiation of iPSCs into CMCs}

We generated and characterized iPSCs from adult skin fibroblasts as described in our earlier publication ${ }^{[5]}$. Briefly, iPSCs were cultured in Nutristem medium on a matrigel coated 6-well plate surfaces (BD biosciences) for three days at a seeding density of $10^{6}$ cells/well. When the culture reached $80 \%-$ $90 \%$ confluency, the cells were treated with iPSC medium containing $1 \mu \mathrm{mol} / \mathrm{L}$ GSK inhibitor BIO (Tocris Bioscience) and bFGF (10 ng/mL) for two days followed by RPMI medium containing ascorbic acid $(213 \mu \mathrm{g} / \mathrm{mL})$ supplemented with Wnt inhibitor ( $2 \mu \mathrm{mol} / \mathrm{L}$ of WntC59, Tocris Bioscience) and $0.05 \%$ human serum albumin for subsequent five days. We observed and recorded the beating of CMCs in the cultures from day six onwards.

\section{Flow cytometer analysis}

Flow cytometry analysis was performed to characterize the CMC phenotypes as described earlier by us ${ }^{[15]}$. Briefly, cells from a six-well plate were harvested and labeled with primary Anti-CTT and Gata4 antibodies as well as the appropriate secondary antibodies. The relevant isotypes served as negative controls. Cells were washed three times with FACS buffer, resuspended in $0.5 \mathrm{~mL}$ PBS, and loaded into the flow cytometer (FACSCalibur, BD Biosciences). Data were analyzed by using FlowJo software (Tree Star, Ashland, OR, USA).

\section{Immunofluorescence staining}

Protein expression was evaluated by immunofluorescence staining as previously described by us ${ }^{[16,17]}$. Immunofluorescence imaging utilized either a confocal or wide field epifluorescence microscope (Olympus U-RLF-T, objective magnification: $\times 60)$.

\section{Western blot analysis}

Western blot analyses of Oct4, Sox 2 and Nanog proteins were performed in iPSC cells as described before ${ }^{[16,17]}$ using antibodies purchased from Cell Signaling Technology, USA.

\section{Calcium imaging}

Intracellular free $\mathrm{Ca}^{2+}$ was measured in cells plated in dishes with glass-coverslip bottoms (TPG dish, ThermoFisher, Waltham, PA, USA) and pre-incubated in medium containing a mixture of $10 \mu \mathrm{mol} / \mathrm{L}$ calcium green-AM in $0.4 \%$ pluronic F127 (ThermoFisher) for $30 \mathrm{~min}$. Cells were then washed to remove unincorporated calcium green, and imaged with a $\times 40$ objective of a Nikon TE2000U confocal microscope, equiped with a temperature controlled enclosure maintained at $37^{\circ} \mathrm{C}$. Images were obtained at $1 \mathrm{~s}$ intervals with a $488 \mathrm{~nm}$ Spectra 
Physics (Mountain View, CA, USA) laser. Emitted fluorescence was recorded with a 515/30 nm band pass filter and transmitted light was collected separately to obtain a bright field image.

\section{Particle image velocimetry (PIV) method}

We characterized cell contractility kinetics using our optical flow/PIV method ${ }^{[5,18]}$. High frame rate (10 frame/s) image sequences were recorded using a $\times 20$ objective and a cooled digital CCD camera (Olympus DP72) mounted on an inverted microscope (Olympus TH4-100), equipped with a temperature controlled stage. The cultures were kept at $37^{\circ} \mathrm{C}$ using the heated stage during 2-3 min long imaging sessions. CMC cultures were kept in cell culture incubators between imaging sessions.

A movement pattern (velocity field) captured on a pair of images was extracted by a two-stage predictor-corrector method described by us earlier ${ }^{[18-20]}$. Briefly, instead of relying on frame-to-frame displacements, we identify reference frames in which CMCs are in the relaxed phase. We then characterized each frame of the recording using displacement vectors that are calculated relative to the appropriate reference frame. Beat patterns of individual clusters were obtained by the vectorial average of displacement vectors within a 100-pixel wide area around the center of the cluster. The average difference between the maxima and minima of the beat pattern yield the amplitude of the beating center. Characteristic raise times were obtained as the time required the average beat waveform raises from $20 \%$ to $80 \%$ of the peak amplitude. Similarly, decay times were obtained as the time required for the average wave form to drop from $80 \%$ to $20 \%$ of the peak amplitude. In this study, we further analyzed beat patterns by Fourier spectrum analyses and presented power density spectra as heat maps. The source code of the analysis software is available at http://github.com/aczirok/piv-div

\section{Fourier and power density spectra}

Fourier spectra, $F(f)$, were calculated from beat patterns using the discrete Fourier transform algorithm. Power density spectra, $P(f)$, were obtained as $P(f)=|F(f)|^{2}$, and were used to indicate periodicity within the signal in the form of peaks at the corresponding frequencies. If the analyzed signal is not a pure sine wave, the appearance of harmonics are expected at integer multiples of the fundamental frequency $f(2 f, 3 f$, etc $)$. The magnitude of a peak in the power spectrum indicates the amplitude of the signal oscillating with the corresponding frequency. Unfortunately, the beat pattern is not stationary over the entire observation period, our optical flow analysis often yields an increasing baseline. This artifact reduces the reliability of the Fourier components corresponding to very low frequencies. Hence, frequencies around zero, representing signals with periods longer than quarter of the duration of the recording, were excluded from our analysis.

Power density spectra of multiple beat patterns, typically recorded at progressively later time intervals, are presented as heatmaps. In these images, the horizontal and vertical axes indicate the individual samples and the frequencies, respectively. Amplitudes are color coded according to a linear scale. Power density heat map plots thus allow an unbiased, quantitative characterization of changes in beat frequencies.

\section{Modeling}

The membrane potential and ionic currents were modeled using the OpenCOR (www.opencor.es) platform. The CellML model of the McAllister-Noble-Tsien Model $B^{[21]}$ was obtained from models.cellml.org. Activation or blockage of ion channels was represented by increasing or decreasing the affected ion current by $50 \%$ compared to the published (baseline) scenario.

\section{Statistical analysis}

All experiments were repeated at least 3 times. Results are presented as mean \pm SEM. Comparisons were performed by ANOVA (GB-STAT; Dynamic Microsystems) or $x^{2}$ test for percentages. All tests were 2-sided, and probability values less than 0.05 were considered as statistically significant.

\section{Results}

\section{Characterization of day 14 human iPSC-derived cardiomyocytes} Human iPSC-derived cardiomyocytes (iCMCs) were generated using culture conditions conducive for cardiac differentiation as described by us earlier ${ }^{[5]}$. From in vitro day six onwards, iCMCs exhibited spontaneous beating which can be observed by phase contrast microscopy. Day 14 iCMCs were harvested to evaluate the presence of CMC-specific proteins by flow cytometry analysis. We found that more than $85 \%$ of cells are positive for cardiac specific markers, either cardiac troponin $\mathrm{T}$ (CTT) or $\mathrm{Nkx2.5}$ (Figure 1A). To characterize the maturation process of $\mathrm{iCMCs}$, we investigated the presence of cardiac-specific proteins in cultures of various in vitro age by Western analysis. A comparison of samples from day 7, 14 and $30 \mathrm{iCMC}$ cultures revealed a substantial and progressive increase in the amount of cardiac proteins of hyperpolarization activated cyclic nucleotide gated potassium channel 4 (HCN4), alpha sarcomeric actin (a-SA) and cardiotroponin T (CTT) by day 30 (Figure 1B).

To characterize which CMC types develop within the cardiac-specific differentiation culture, we have performed immunofluorescence analysis identifying myosin light chain variants specific for atrial (MLC2a) and ventricular (MLC2v) cells. Our immunofluorescence staining data showed that the population of day 14 iCMCs were heterogeneous, consisting of both MLC2a positive and MLC2v positive cells (Figure 1C). The immunofluorescence data were further supported by flow cytometry analysis indicating that $86.3 \%$ of cells were positive for either MLC2a or MLC2v (Figure S1A). The quantification of flow cytometry data revealed that the differentiation protocol was more supportive for the differentiation towards ventricular cardiomyocytes (Figure S1B).

In addition to the presence of appropriate molecular markers, transmission electron microscopic image analysis of day 14 iCMCs revealed a well-defined and mature myofibrillar pat- 
A

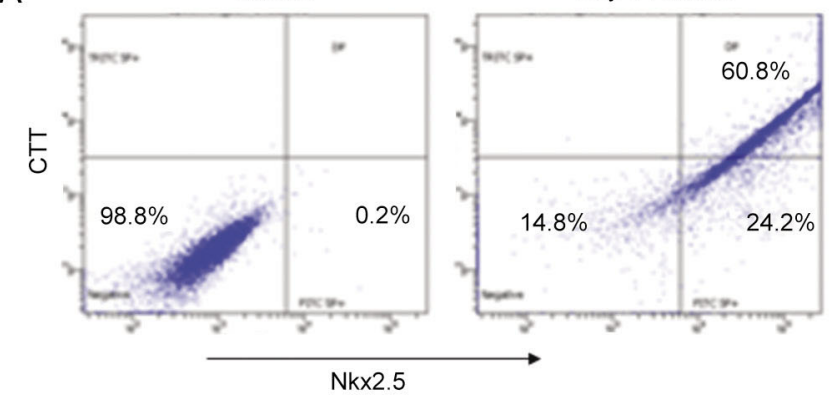

C
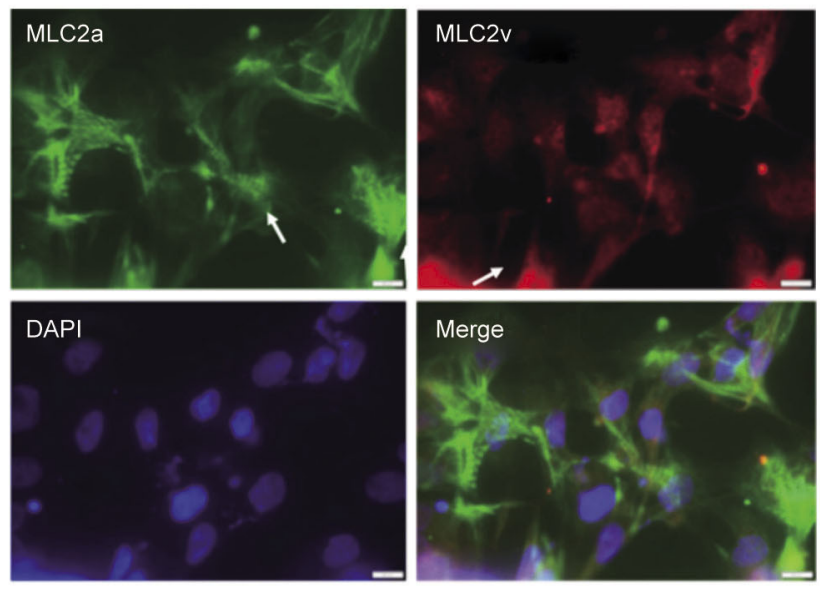
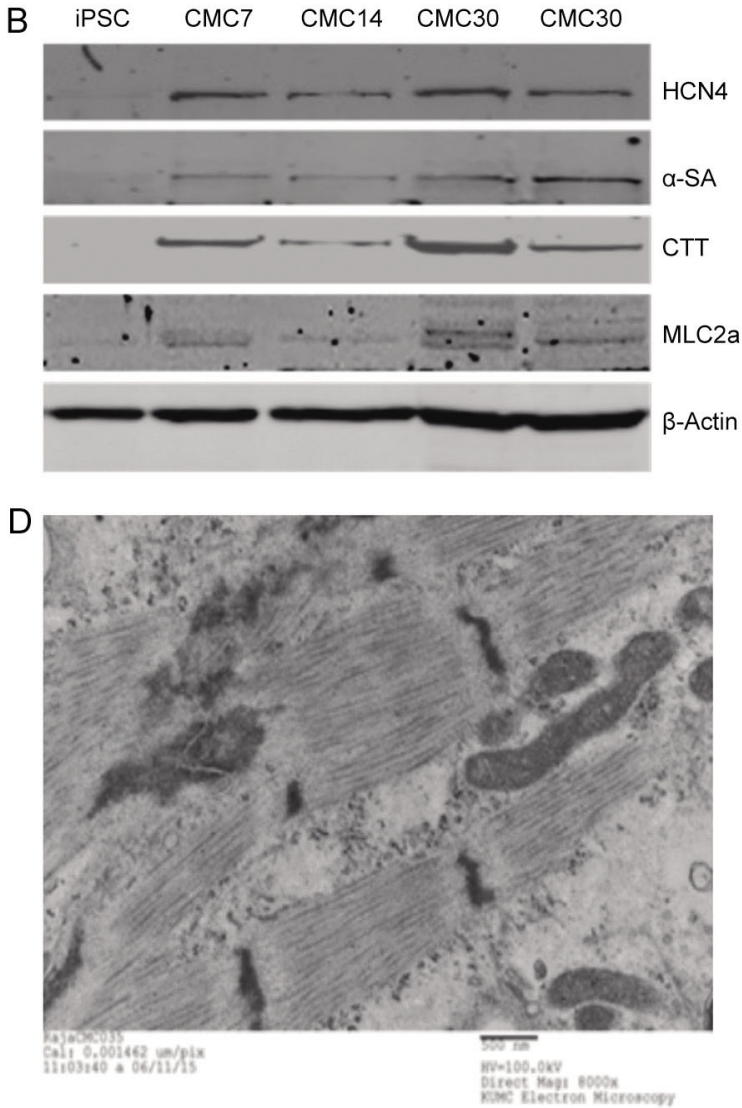

Figure 1. Characterization of iPSC-derived cardiomyocytes (iCMCs). (A) Flow cytometry analysis on day 14 iCMCs show $85.8 \%$ of cells are stained positive for cardiac specific proteins either Nkx2.5 or CTT. (B) Western analysis data showed an increased expression of cardiac specific proteins HCN4, $\alpha$-SA and CTT. The $\beta$-actin serves as a loading control for proteins. (C) Immunofluorescence analysis for the expression of MLC2a (green) and MLC2v (red) represents the presence of atrial and ventricular CMCs in the culture, DAPI (blue) used for staining nuclei. (D) The maturity of day 14 CMCs is indicated by transmission electron micrographs (TEM) depicting a high density of organized and aligned sarcomeres along with the myofibrils. Scale bars $10 \mu \mathrm{m}$ for immunostaining images and $500 \mathrm{~nm}$ for TEM.

tern of sarcomeres (Figure 1D). Functional CMCs also require the expression of appropriate $\mathrm{Ca}^{2+}, \mathrm{K}^{+}$and $\mathrm{Na}^{+}$channels ${ }^{[22]}$, their presence was verified by immunofluorescence analysis (Figure S2). Overall, these data suggest that by day 14 iCMC cultures contain functional cardiomyocytes, and cultures of this age were subjected to our optical beat pattern analysis.

\section{Contactless cardiac contractility analysis}

To characterize chronotropic alterations in CMC beat frequency, we recorded the spontaneous beating activity of the same iCMC cluster at various time points using video microscopy and a temperature controlled stage. The first recording, made one hour after refreshing the culture medium, served as a baseline. Then, we administered the drug into the culture medium and recorded beat activity periodically. One of these recordings took place $2 \mathrm{~h}$ after the addition of the drug, and we used this recording to establish the steady-state effect of the compound (see below). After a treatment window of 2-4 h, the drug containing medium was removed, the cells were washed with PBS and the culture dish was filled with fresh medium. Finally, $18 \mathrm{~h}$ after the wash, the recovered
iCMC culture was recorded again to evaluate the reversibility of the pharmacological effects.

Recordings were later analyzed by our optical flow/PIV technique $^{[5]}$ yielding beat patterns-tissue displacement as a function of time, measured relative to a resting reference state. Beat patterns consist of a sequence of peaks, each corresponding to a contractile event. The duration of the raising and falling slopes of the peaks thus estimate the length of the electrophysiological plateau and repolarization phases, respectively (Figure S2A, B). To extract beat frequency, beat patterns were subjected to Fourier analysis, and the dominant beat frequencies were visualized on power density spectrograms. Periodicity within the beat pattern is indicated in the power spectra by a peak at the corresponding frequency. The magnitude of a peak is related to the amplitude of the signal oscillating with the corresponding frequency (Figure S2C). Thus, CMC beat patterns are characterized by two numbers, the spontaneous beat frequency (SBF) and spontaneous beat amplitude (SBA). Power spectra of multiple beat patterns, typically recorded at progressive time intervals, are presented as heat maps (Figure S2D).

For each drug, we compare the baseline with the $2 \mathrm{~h}$ post- 
A
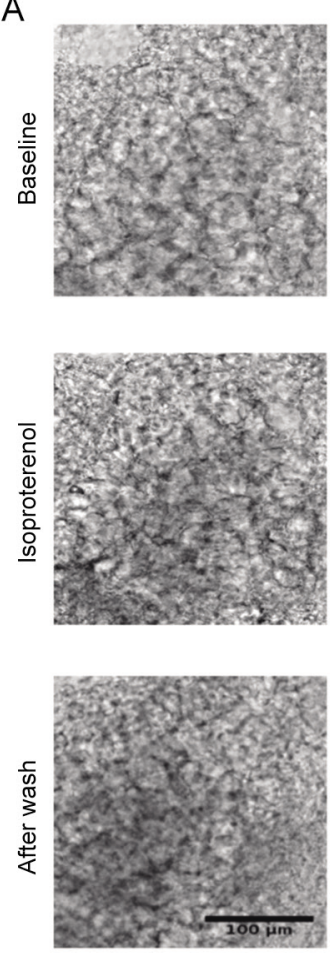

B
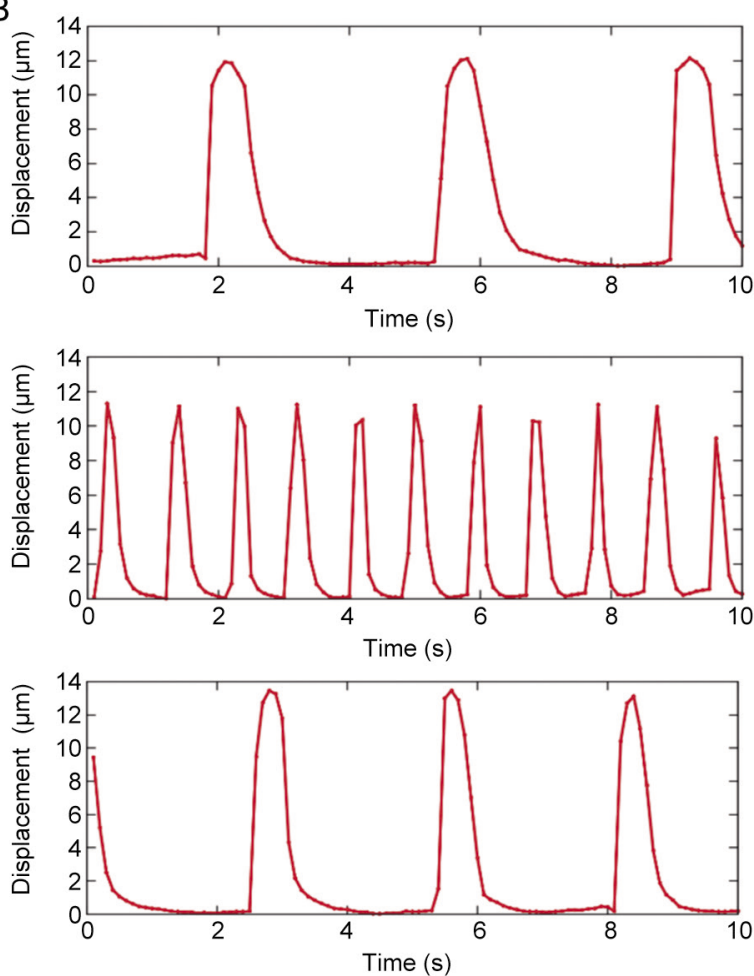

C
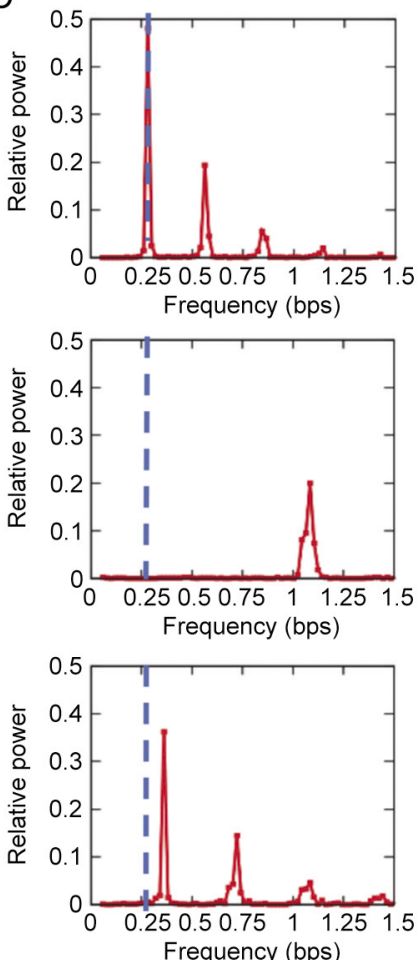

D

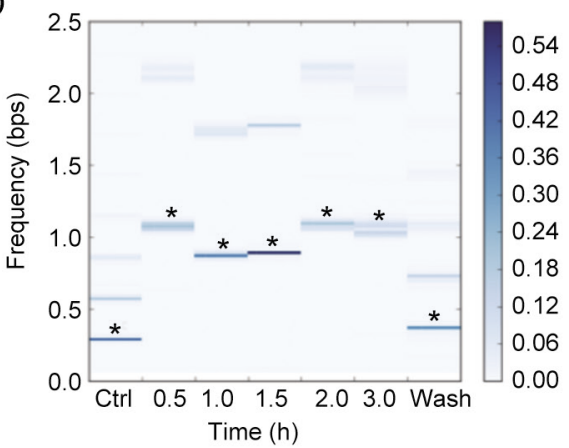

E

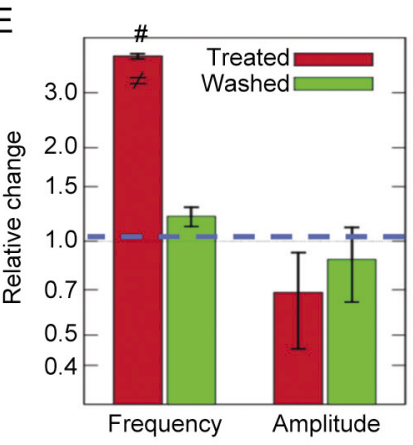

F

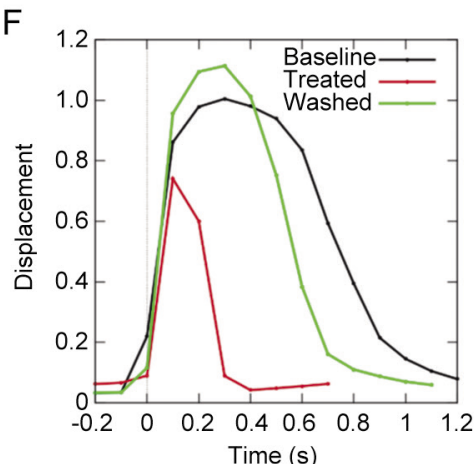

Figure 2. Chronotropic effects of isoproterenol in day 14 iCMCs. (A) Phase contrast microscopic image of functional iCMCs, before, during and after treatment with $800 \mathrm{nmol} / \mathrm{L}$ isoproterenol. (B) Spontaneous beat patterns expressed as displacement vs time. (C) Fourier power spectra of beat patterns indicate the dominant beat frequency (SBF) as a peak. The increase in SBF is manifested as a shift of the dominant peak towards higher frequencies (right) from the baseline, indicated by the vertical dashed line. (D) Power spectrograms of consecutive recordings shown as a heat map, and base frequencies are marked by asterisks. (E) Pooled responses of $n=3 \mathrm{iCMC}$ cultures to $800 \mathrm{nmol} / \mathrm{L}$ isoproterenol (red) and to a subsequent recovery period (green). Beat frequencies (NBF) and amplitudes (NBA) are normalized to the values obtained of the same cultures before treatment (horizontal dashed line). ${ }^{\#} P<0.03$. (F) Average waveform of the beat pattern indicates a shortening of the rise and decay times of iCMC contractility. Scale bars $100 \mu \mathrm{m}$.

treatment and the $18 \mathrm{~h}$ post-wash data. To reduce the effect of culture variability, the SBF and SBA values were normalized with the corresponding baseline values $\left(\mathrm{SBF}_{0}\right.$ and $\left.\mathrm{SBA}_{0}\right)$, yielding the normalized beat frequency $\left(\mathrm{NBF}=\mathrm{SBF} / \mathrm{SBF}_{0}\right)$ and normalized beat amplitude (NBA=SBA$/ \mathrm{SBA}_{0}$ ) measures, respectively. To establish the quality and reproducibility of our drug screening procedure, we performed our experimental protocol with adding only a treatment vehicle, DMSO. Comparison of the baseline, $2 \mathrm{~h}$ post-treatment and $18 \mathrm{~h}$ postwash frequencies indicate only minor, statistically insignificant differences. Normalized frequency and amplitude values are compared to 1 , which is represented by the horizontal dotted line $(\mathrm{NBF}=1, \mathrm{NBA}=1$, Figure S2E). Thus, our optical and computational analysis of iCMC cultures indicates that the spontaneous beat frequency or amplitude was stable during the time period selected for chronotropic analysis. The average raise and decay times of the beat patterns were obtained from $(n=8)$ averaged waveforms yielding $0.12 \pm 0.02$ and $0.20 \pm 0.05 \mathrm{~s}$, respectively (Figure S2F).

\section{Beta-adrenergic calcium channel regulator}

Our immunofluorescence microscopic image analysis showed 


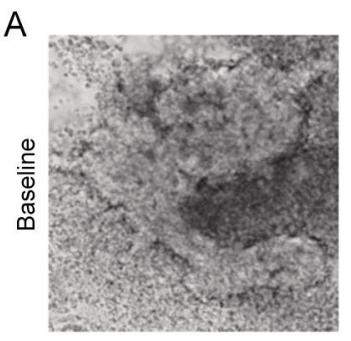

B
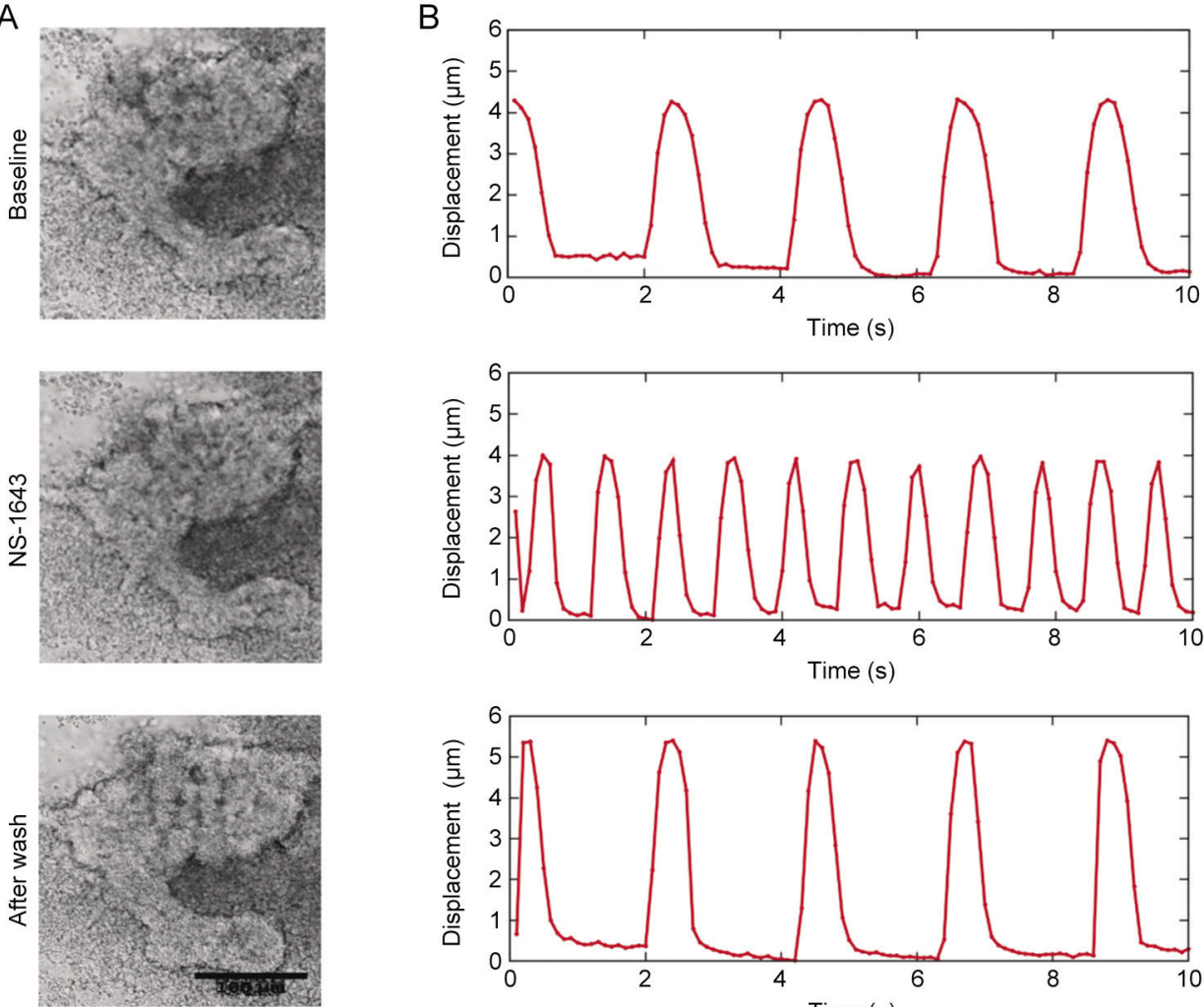

D

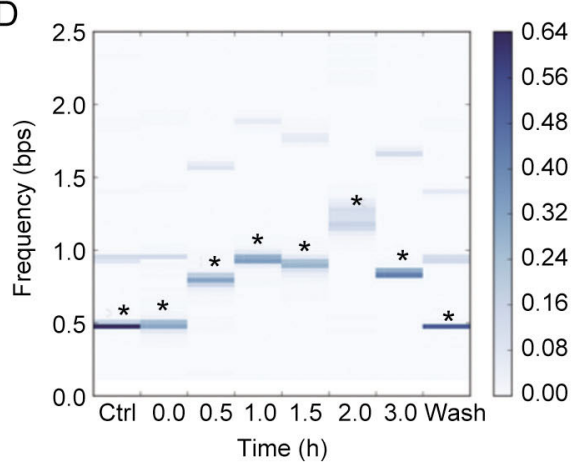

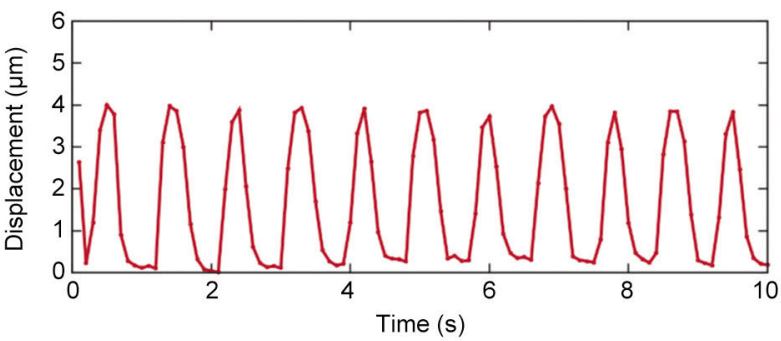

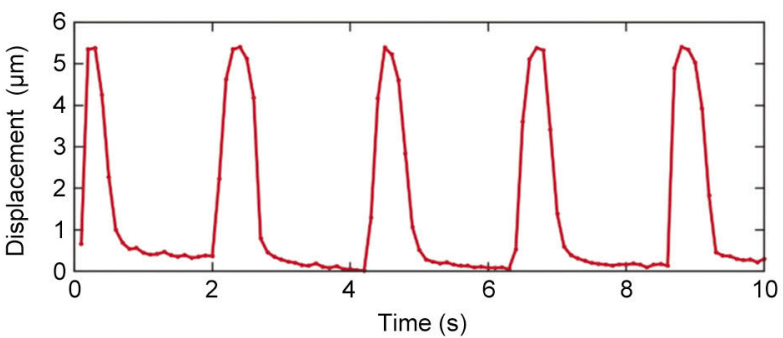

E

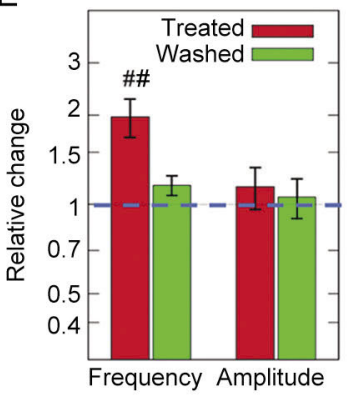

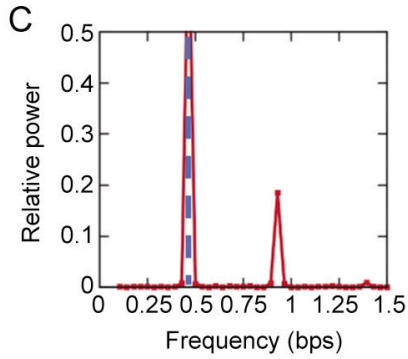
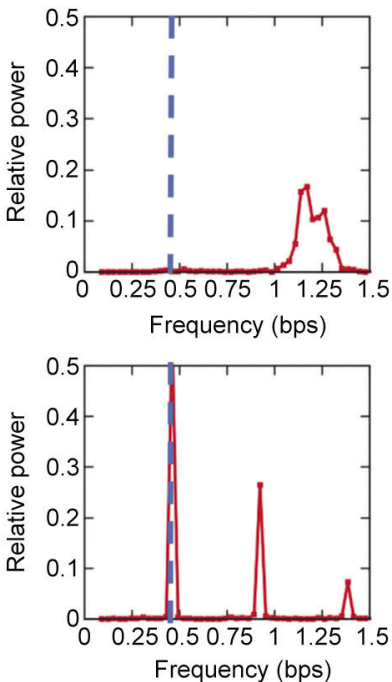

$\mathrm{F}$

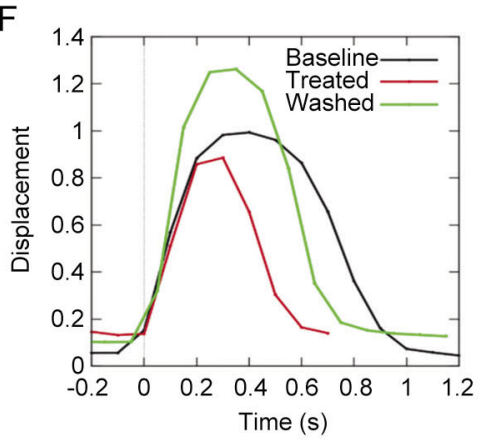

Figure 3. Chronotropic effects of NS1643, a potassium channel activator, in day 14 iCMCs. Panels (A-F) represent the same images and statistical measures as in Figure 2. (A) Phase contrast micrographs of iCMCs, before, during and after treatment with 60 nmol/L NS1643. (B) Spontaneous beat patterns calculated from time-lapse recordings. (C) Fourier power spectra of beat patterns indicate the increase in SBF as a shift of the dominant peak towards higher frequencies (right). (D) Heat maps of power spectrograms, with base frequencies marked by asterisks. (E) Pooled responses of $n=3$

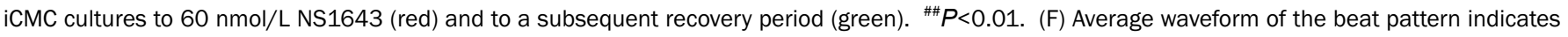
a shortening of the decay time of iCMC contractility. Scale bars $100 \mu \mathrm{m}$.

that cultured iCMCs expressed $\mathrm{Ca}^{2+}$ channels encoded by the CACNA1S gene that are required for the polarization-repolarization cycles in cardiac muscle (Figure S3A). Isoproterenol $(800 \mathrm{nmol} / \mathrm{L})$, the $\beta$-adrenergic agonist influencing intracellular $\mathrm{Ca}^{2+}$ dynamics, induced a substantial and sustained increase in beating frequency without triggering arrhythmias (Figure 2A, B; Supplemental Movie SM1). Fourier analysis clearly indicates a shift in the SBF (Figure 2C, dotted line). Power spectra of consecutive recordings are shown as a heat map (Figure 2D). The SBF for each sample is marked by an asterisk quantifying the gradual, sustained increase in SBF. The reversibility of the isoproterenol effect is indicated by the SBF returning to the baseline value after drug removal (Figure 2A, E). In summary, $800 \mathrm{nmol} / \mathrm{L}$ isoproterenol in our assay exerts a substantial but reversible increase in SBF $(\mathrm{NBF}=3.93 \pm 0.07, P<0.03)$ and a weaker, not significant decrease in SBA (NBA=0.69 $\pm 0.23, P=0.2)$. The increased SBF reflected significant, $30 \%$ and $70 \%$ decreases in the raise and decay time of the average beat pattern, respectively $(P<0.01$, Figure $2 \mathrm{~F})$. Therefore, iCMCs exhibit a physiologic chronotropic response 
A
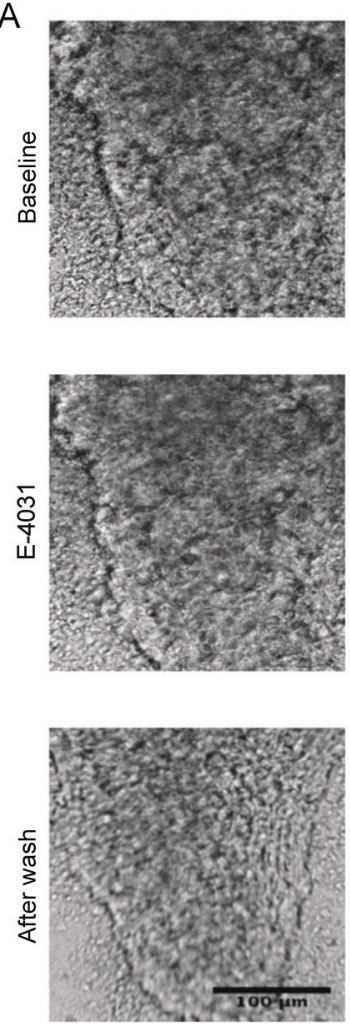

B
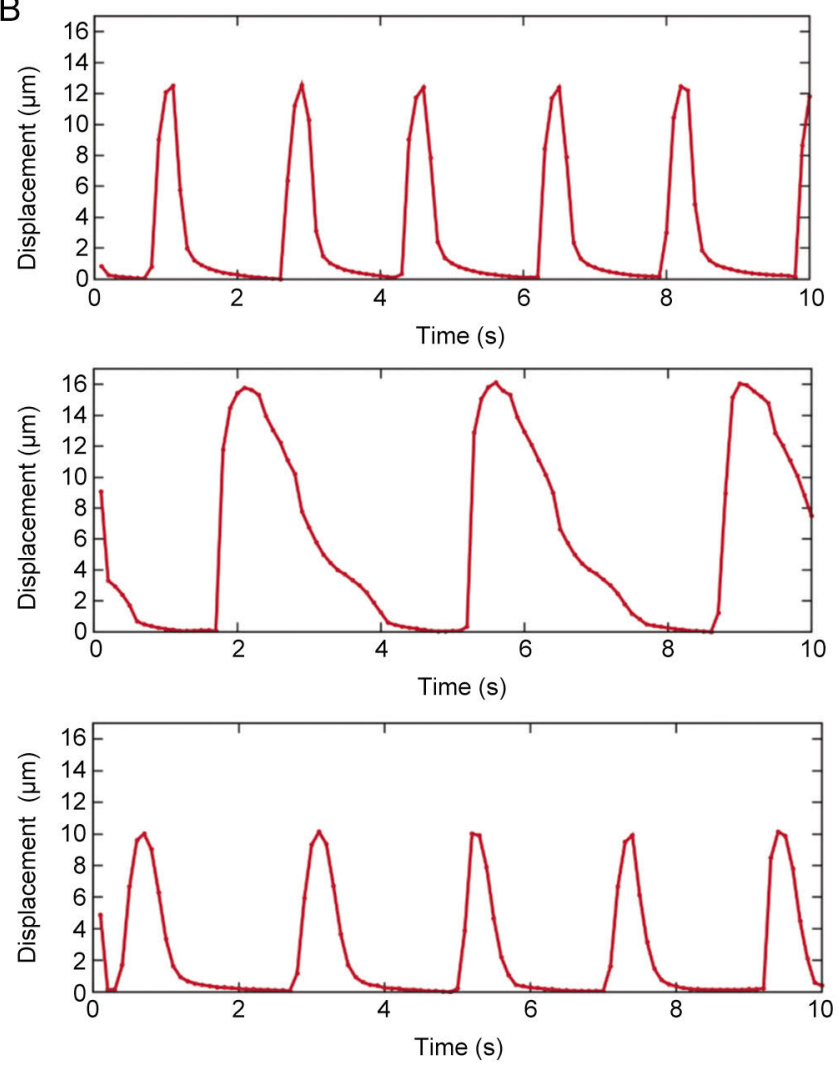

C
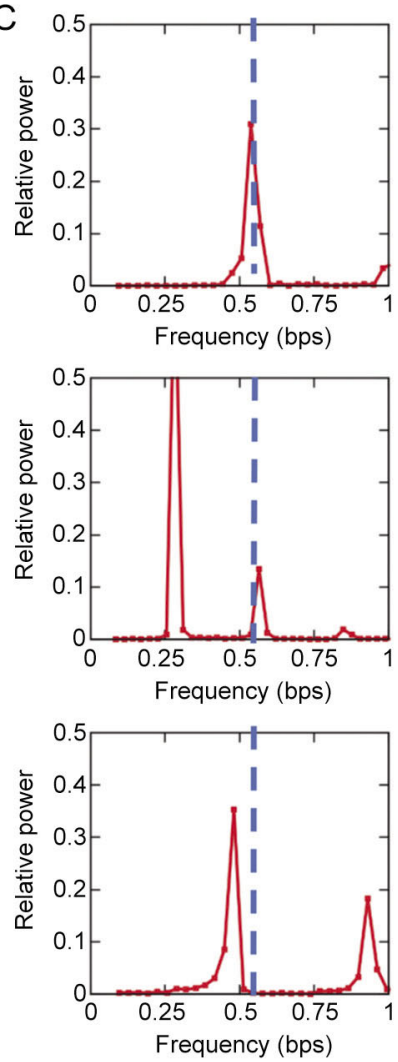

D

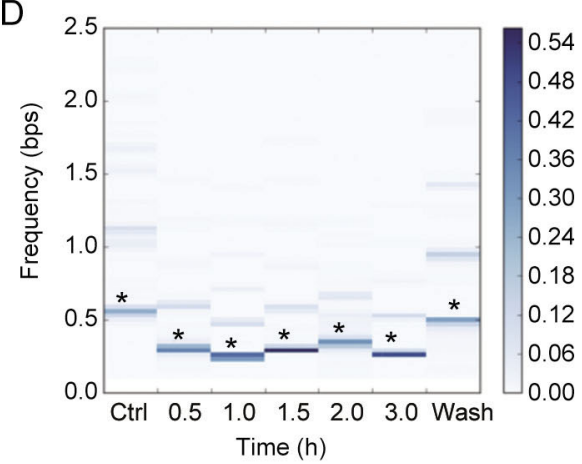

E

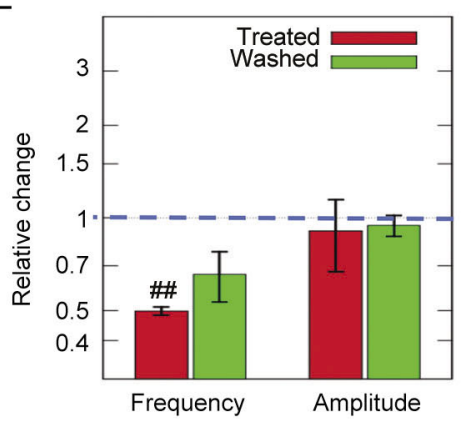

F

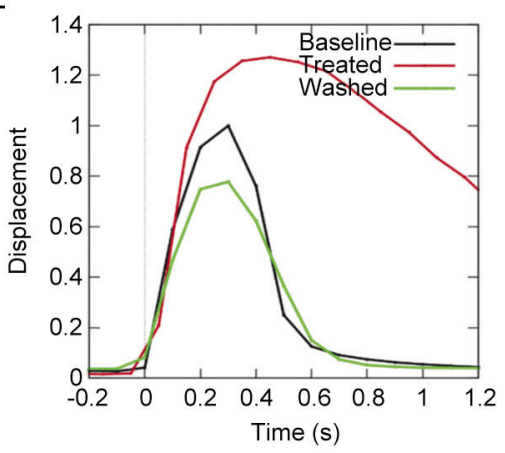

Figure 4. Chronotropic effects of E4031, potassium channel blocker, on day 14 iCMCs. Panels (A-F) represent the same images and statistical measures as in Figure 2. (A) Phase contrast micrographs of iCMCs, before, during and after treatment with $500 \mathrm{nmol} / \mathrm{L}$ E4031. (B) Spontaneous beat patterns calculated from time-lapse recordings. (C) Fourier power spectra of beat patterns indicate the decrease in SBF as a shift of the dominant peak towards lower frequencies (left). (D) Heat maps of power spectrograms, with base frequencies marked by asterisks. (E) Pooled responses of $n=3$ iCMC cultures to $500 \mathrm{nmol} / \mathrm{L}$ E4031 (red) and to a subsequent recovery period (green). ${ }^{\# \#} P<0.01$. (F) Average waveform of the beat pattern indicates a substantially increased decay time of iCMC contractility. Scale bars $100 \mu \mathrm{m}$.

to an adrenergic stimulus.

\section{Voltage-gated potassium channel regulators}

Voltage-gated potassium channels (Kvs) were indispensable regulator to generate action potential currents for spontaneous beat pattern in $\mathrm{CMCs}^{[23]}$. Kvs channels are expressed by iCMCs as immunofluorescence analysis reveals (Figure S3B). In this study, we have used the small molecule Kvs activator NS1643 ${ }^{[24]}$ and Kvs blocker E4031 ${ }^{[25]}$ to study their chrono- tropic and ionotropic effects in human iCMC cultures.

The SBF of iCMCs treated with $60 \mathrm{nmol} / \mathrm{L}$ NS1643 increased significantly to $\mathrm{NBF}=1.97 \pm 0.29(P<0.03)$ by $2 \mathrm{~h}$ after the addition of drug (Figure 3, Supplemental Movie 2). The SBA remained unchanged within measurement error $(\mathrm{NBA}=1.15 \pm 0.18$, $P=0.25$ ). Beat patterns (Figure $3 \mathrm{~A}-\mathrm{E}$ ) indicate that the increase in SBF involves a dramatic shortening of both the relaxation and resting phases of the $\mathrm{CMC}$ contractile cycle. The positive chronotropic effect induced by NS1643 was reversible: 16 h 
A
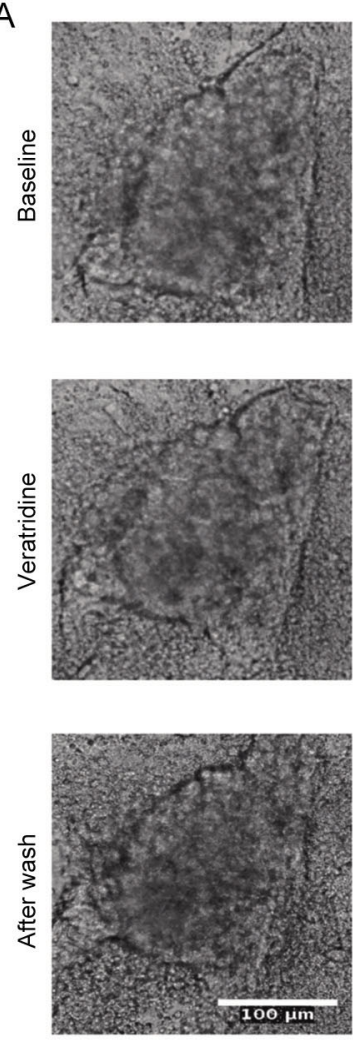

B
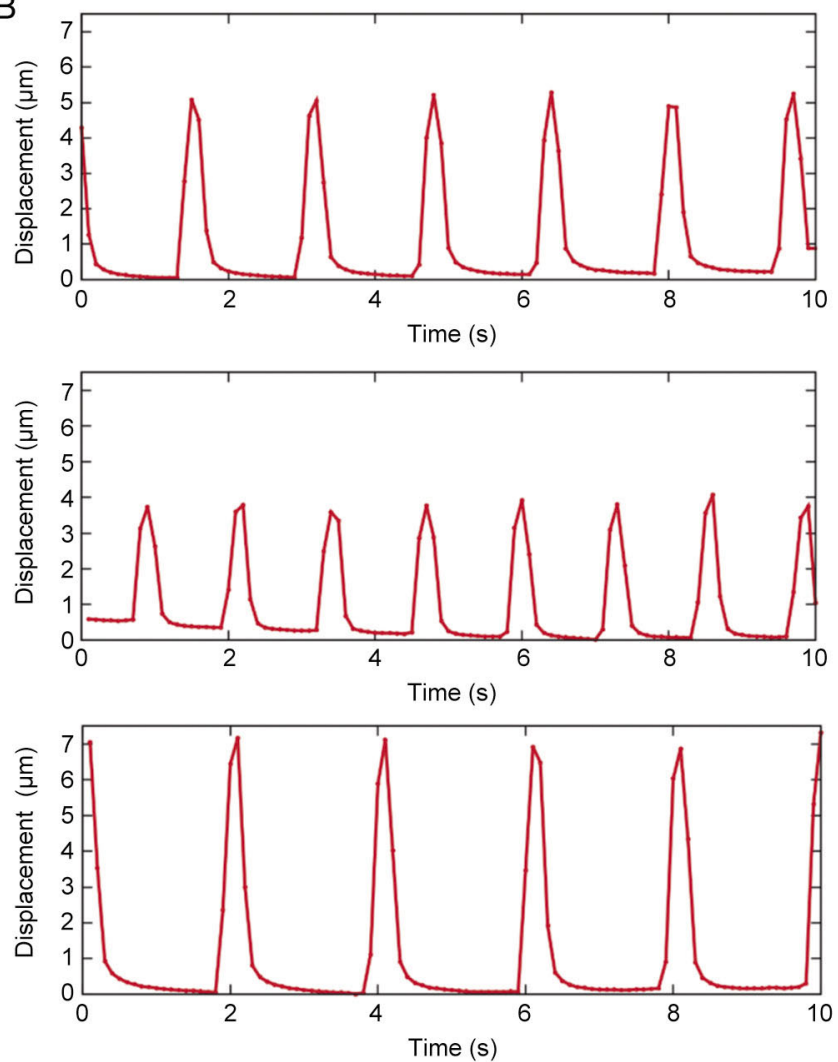

C
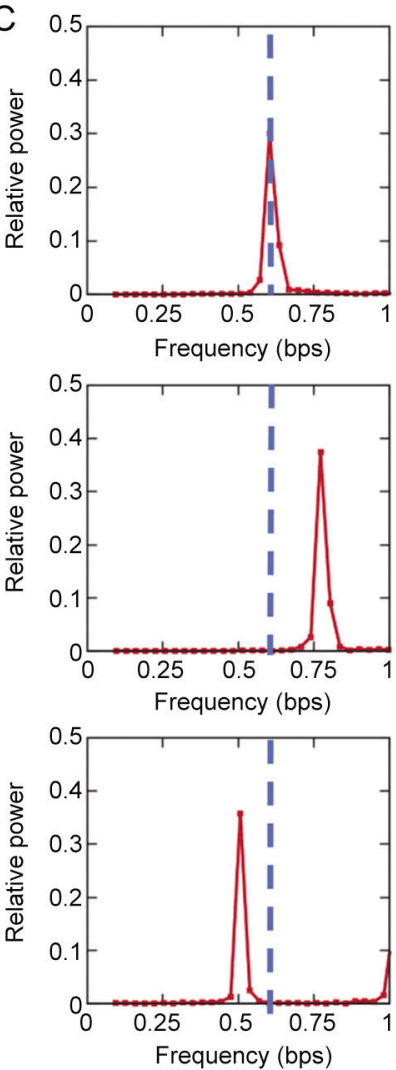

D

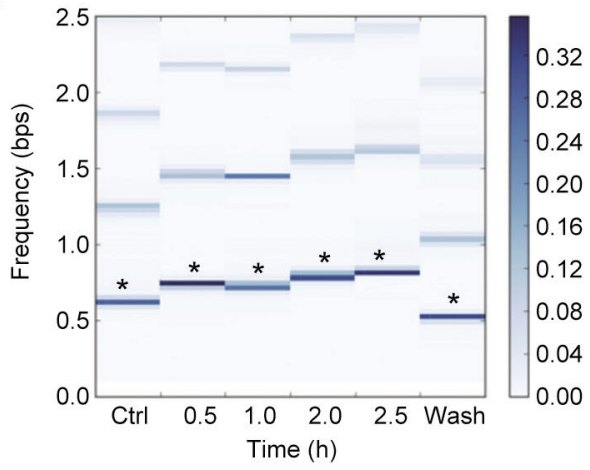

E

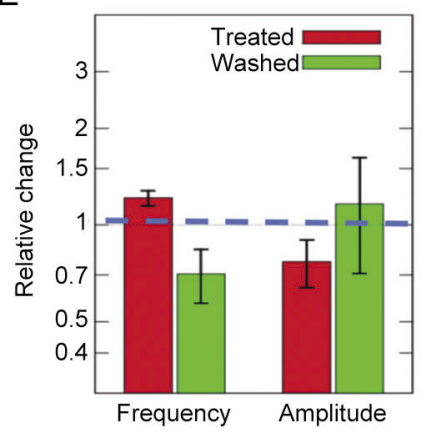

F

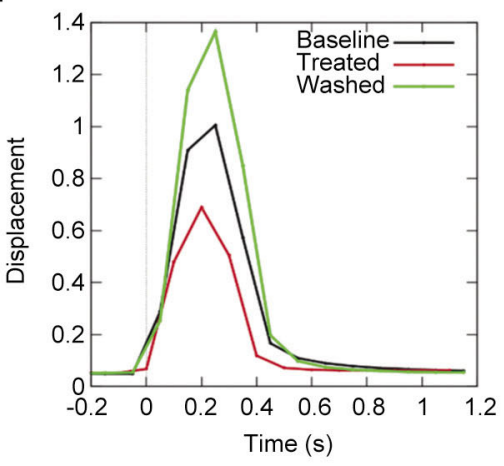

Figure 5. Chronotropic effects of the sodium channel activator veratridine in day 14 iCMCs. Panels (A-F) represent the same images and statistical measures as in Figure 2. (A) Phase contrast micrographs of iCMCs, before, during and after treatment with $50 \mathrm{nmol} / \mathrm{L}$ veratridine. (B) Spontaneous beat patterns calculated from time-lapse recordings. (C) Fourier power spectra of beat patterns indicate the increase in SBF as a shift of the dominant peak towards higher frequencies (right). (D) Heat maps of power spectrograms, with base frequencies marked by asterisks. (E) Pooled responses of $n=3 \mathrm{iCMC}$ cultures to $50 \mathrm{nmol} / \mathrm{L}$ veratridine (red) and to a subsequent recovery period (green). The increase in SBF is not significant $(P=0.096)$. (F) Average waveform of the beat pattern does not indicate a substantially altered raise or decay time of iCMC contractility. Scale bars $100 \mu \mathrm{m}$.

after removing the drug, both SBF and SBA values returned to the baseline level (Figure 3D-F).

The hERG inhibitor, E4031, is expected to extend the repolarization phase of the action potential in human $\mathrm{CMCs}^{[26]}$. Accordingly, $500 \mathrm{nmol} / \mathrm{L}$ E4031 decreased the SBF to $\mathrm{NBF}=0.49 \pm 0.02(P<0.01$, Figure 4, Supplemental Movie 3). Beat patterns (Figure 4A-E) indicated that the decreased $\mathrm{SBF}$ is mainly due to an 8 -fold longer decay time $(1.9 \pm 0.1 \mathrm{~s}$, $P<0.001$, Figure $4 \mathrm{~F})$ : the contraction and resting phases appear to be unchanged. The SBA was not affected by E4031 as $\mathrm{NBA}=0.91 \pm 0.24(P=0.36)$. After removing the drug, the SBF increased, but tended to remain below the baseline level with $\mathrm{NBF}=0.65 \pm 0.1(P=0.05$, Figure 4D, E). For E4031 we also compared our optical analysis with a more traditional calcium transient imaging method, and obtained a similar response $(\mathrm{NBF}=0.6 \pm 0.1$, Figure S4). In cultures of iCMC cells, NS1643 and E4031 exerts potent positive and negative chronotropic effects respectively, without influencing the amplitudes of the 
A
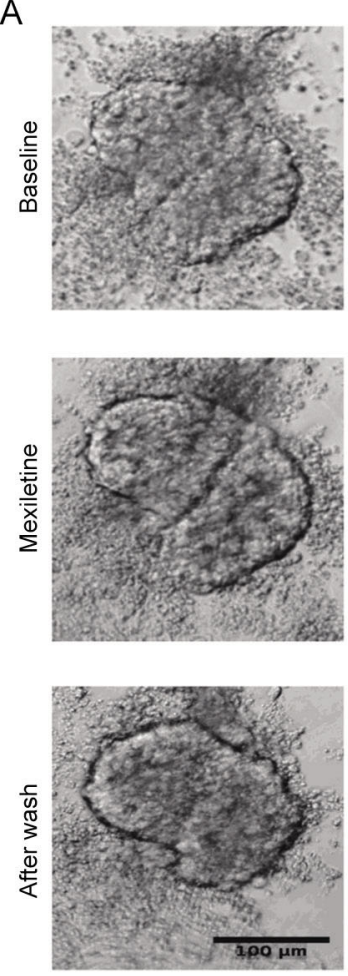

$\mathrm{B}$
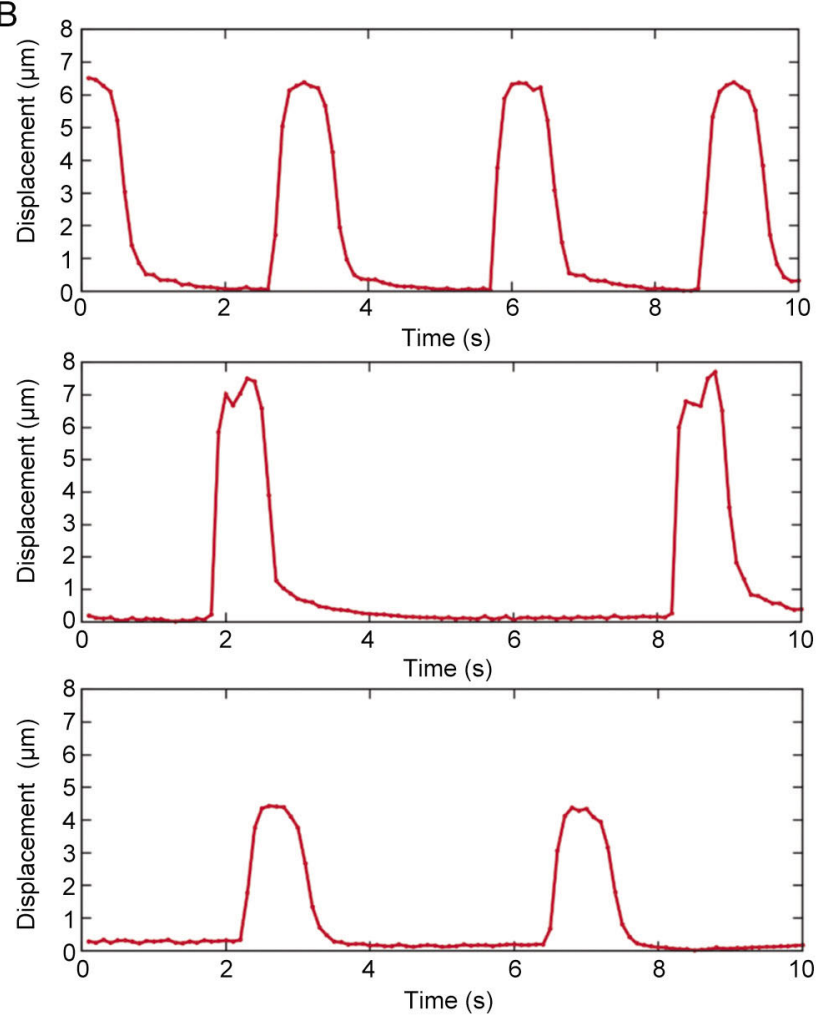

C
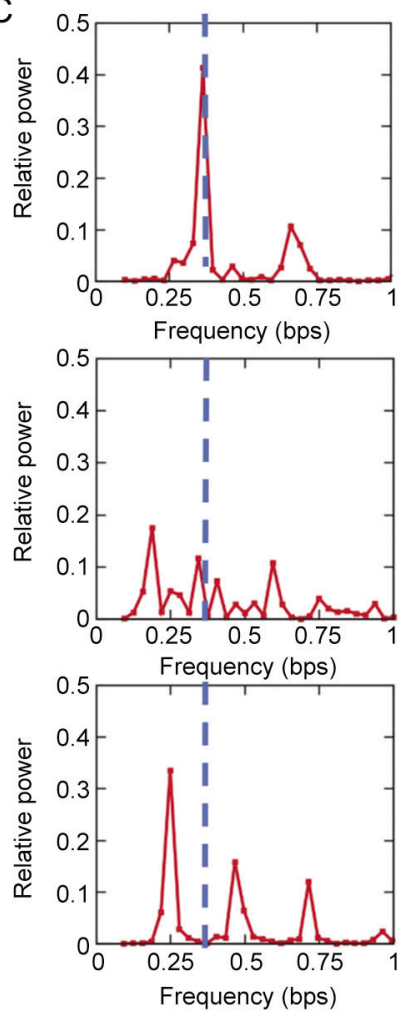

D

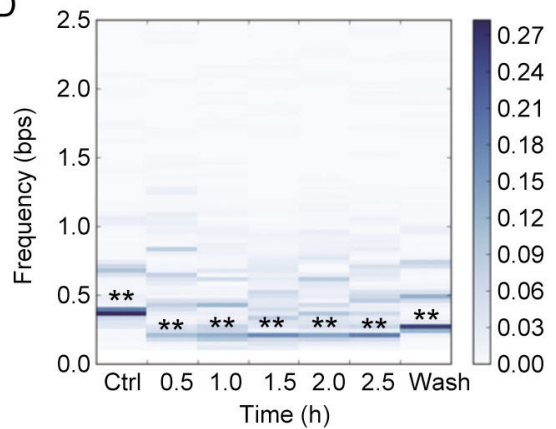

$\mathrm{E}$

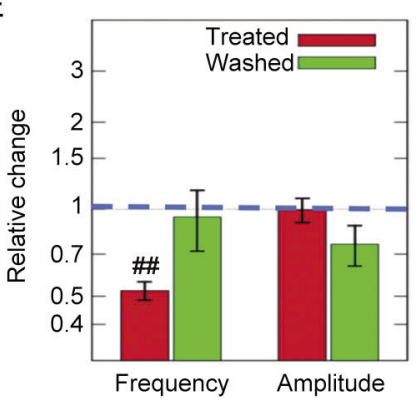

$\mathrm{F}$

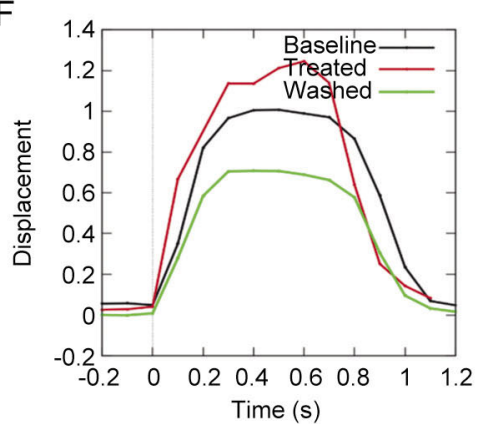

Figure 6. Chronotropic effects of mexiletine, a sodium channel blocker in day 14 iCMCs. Panels (A-F) represent the same images and statistical measures as in Figure 2. (A) Phase contrast micrographs of iCMCs, before, during and after treatment with $100 \mu \mathrm{mol} / \mathrm{L}$ mexiletine. (B) Spontaneous beat patterns calculated from time-lapse recordings. (C) Fourier power spectra of beat patterns indicate the decrease in SBF as a shift of the dominant peak towards lower frequencies (left). (D) Heat maps of power spectrograms, with base frequencies marked by asterisks. (E) Pooled responses of $n=3$ iCMC cultures to $100 \mu \mathrm{mol} / \mathrm{L}$ mexiletine (red) and to a subsequent recovery period (green). ${ }^{\# \#} P<0.01$. (F) Average waveform of the beat pattern indicates an increased raise time of iCMC contractility. Scale bars $100 \mu \mathrm{m}$.

contraction cycle.

\section{Voltage-gated sodium channel regulators}

Voltage gated $\mathrm{Na}^{+}$channels are the third essential components of the action potential oscillator. We therefore, examined the roles of the voltage gated $\mathrm{Na}^{+}$channel activator veratridine ${ }^{[27]}$ and blocker mexiletine ${ }^{[28]}$ on the spontaneous beating properties of human iCMCs. We verified the presence of these proteins by immunofluorescence analysis (Figure S3C).

Our optical beat pattern analysis shows that $50 \mathrm{nmol} / \mathrm{L}$ of the $\mathrm{Na}^{+}$channel activator veratridine significantly increases the SBF to NBF=1.21 \pm 0.07 ( $P=0.096$, Figure 5, Supplemental
Movie 4). Veratridine, however, has no significant effect on SBA $(P=0.164)$. The reversibility of the veratridine effect is indicated when the SBA returns to the baseline value after drug removal (Figure 5D-F). The voltage-gated $\mathrm{Na}^{+}$channel blocker mexiletine, which belongs to class IB anti-arrhythmic drugs, decreased the SBF to NBF $=0.52 \pm 0.04(P<0.01)$ at a concentration of $100 \mu \mathrm{mol} / \mathrm{L}$ (Figure 6, Supplemental Movie 5). Beat patterns (Figure 6A-E) indicate that the decreased SBF is mainly due to a longer relaxation phase and a $50 \%$ increase in the raise time to $0.20 \pm 0.02 \mathrm{~s}(P<0.01$, Figure $6 \mathrm{~F})$. In cultures of iCMCs, thus, the Na channel activator and blocker had a positive and negative chronotropic effect, respectively. 
A

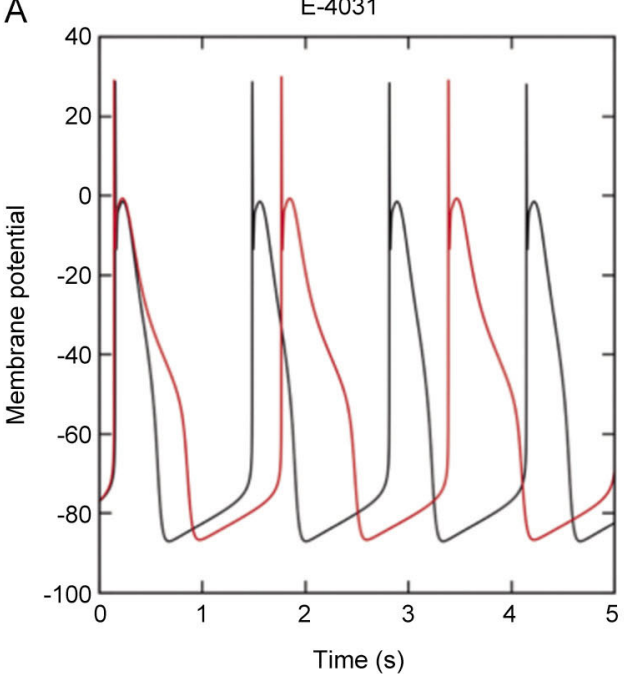

C

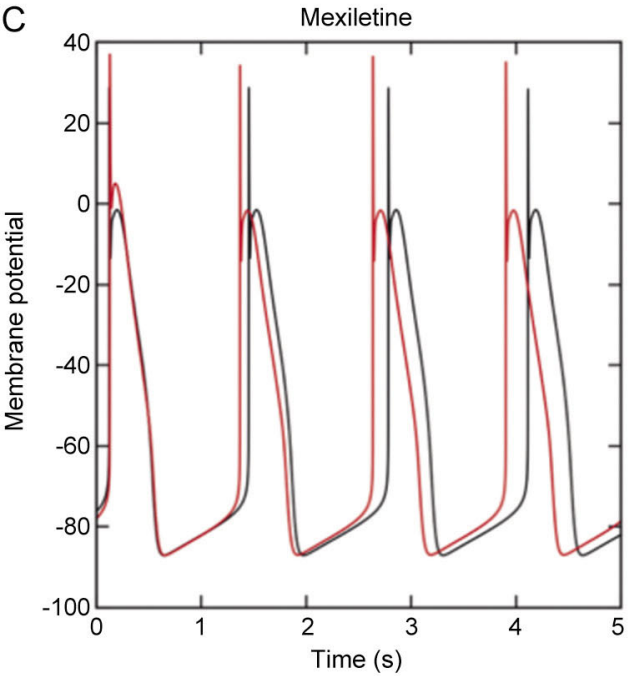

B

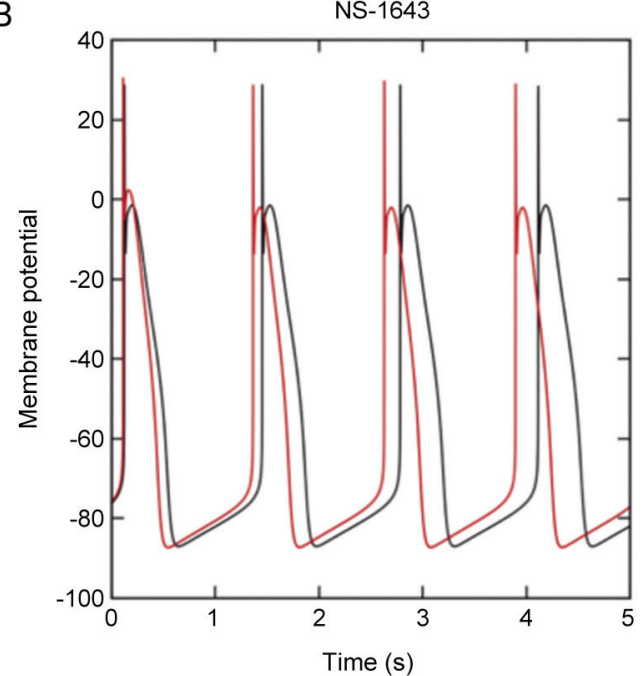

D

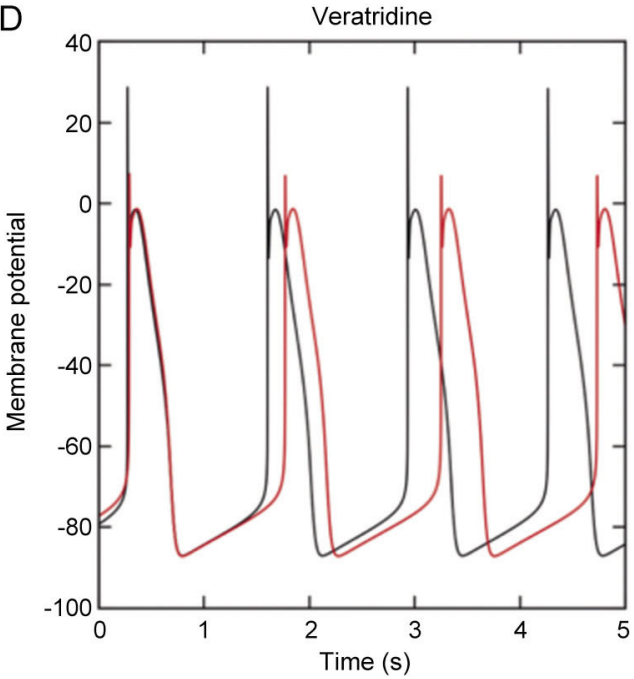

Figure 7. Effects of ion channel modulators on cardiomyocyte spontaneous beating activity, as predicted by the McAllister-Noble-Tsien Model B of Purkinje fibers. We used the OpenCor framework and the CelIML formulation of the model to calculate the time development of the transmembrane potential for the baseline parameters (black graphs) and the appropriate ionic currents modulated by $50 \%$ (red graphs). The effects of E-4031 (A), NS1643 (B), mexiletine (C) and veratridine (D) were represented by decreasing or increasing of $\mathrm{K}^{+}$and $\mathrm{Na}^{+}$currents, respectively. The predicted increase or decrease of beating frequency, albeit not their numerical value, is in good agreement with experimental observations.

\section{Computational model}

To interpret the chronotropic effects of the $\mathrm{Na}$ and $\mathrm{K}$ channel regulators, we implemented the perturbations in a computational model of cardiomyocyte electrophysiology. We used the McAllister-Noble-Tsien Model B of Purkinje fibers ${ }^{[21]}$ as it represents the ion channels relevant for our experiments but lacks additional modeling complexity. In the computational model activation or blockage of ion channels was represented by increasing or decreasing the affected ion current by $50 \%$ compared to the baseline scenario.

According to the Model $\mathrm{B}$, extracellular-directed $\mathrm{K}$ ion currents determine the length of the plateau and repolarization phases. Reducing K currents thus prolong the duration of the plateau and repolarization phases and decrease the frequency of the action potential cycle (Figure 7A). Conversely, increased $\mathrm{K}$ currents lead to a shorter plateau phase and a faster repolarization process, both of which increase the SBF (Figure 7B).

As voltage-gated fast $\mathrm{Na}^{+}$channels are responsible for the depolarization phase through a self-amplifying process, inactivation of a population of $\mathrm{Na}$ channels lengthened the resting phase by delaying the depolarization events (Figure 7C). Due to the underlying positive feedback, once depolarization is initiated, the membrane potential continues to rise rapidly. $\mathrm{Na}$ channels are mostly closed in the repolarization and plateau phases, which are thus not affected by inactivation of Na channels. Persistent activation of Na channels, in contrast, have two opposite effects: by increasing the membrane potential, it promotes an earlier onset of the depolarization phase. The steady influx of $\mathrm{Na}$ ions, however, work against the outflux of 
A
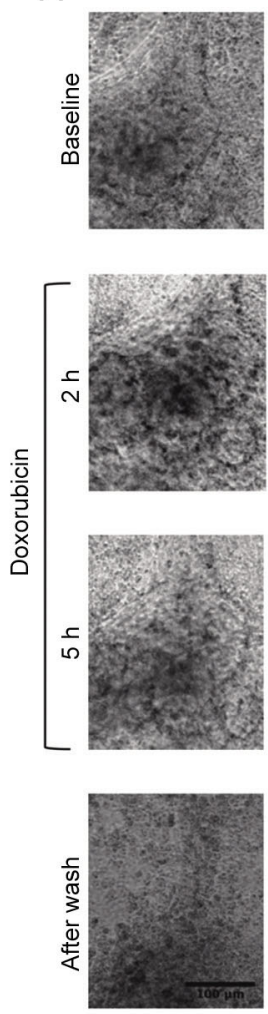

B
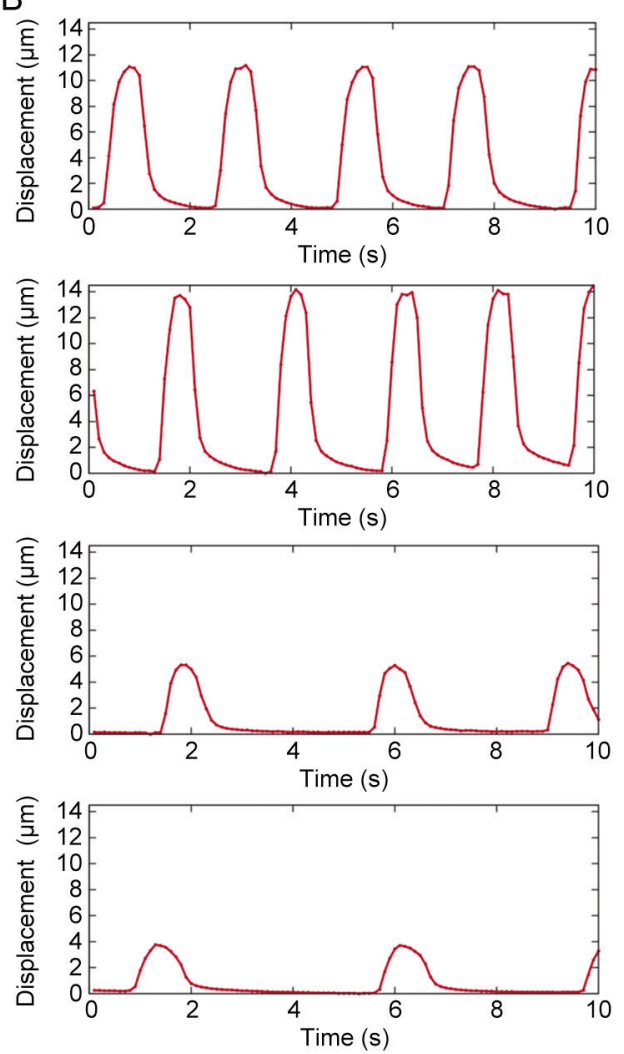

C
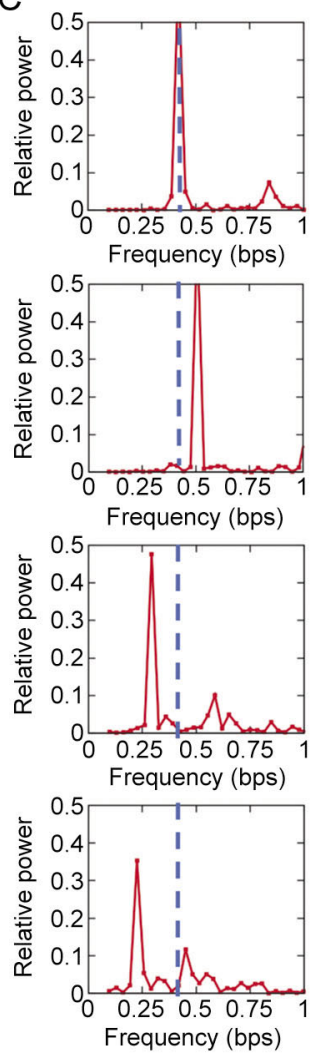

D

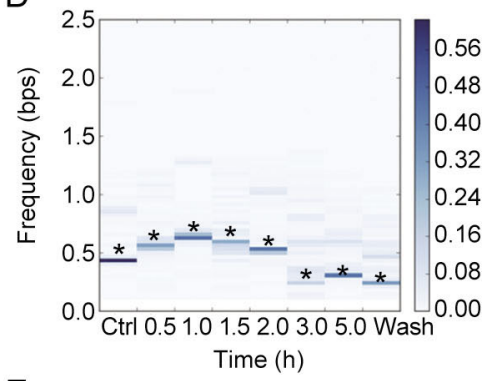

E
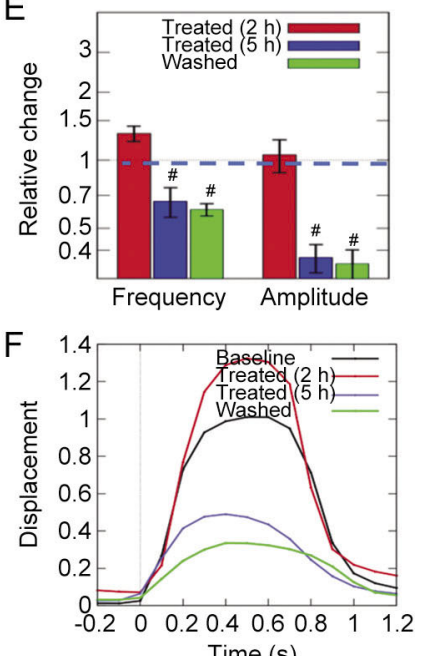

Figure 8. Chronotropic effects of the anti-cancer drug doxorubicin in day 14 iCMCs. Panels (A-F) represent the same images and statistical measures as in Figure 2. (A) Phase contrast micrographs of iCMCs, before, during and after treatment with $10 \mu \mathrm{mol} / \mathrm{L}$ doxorubicin. (B) Spontaneous beat patterns calculated from time-lapse recordings. (C) Fourier power spectra of beat patterns indicate the increase and a subsequent decrease in SBF as a shift of the dominant peak towards higher then lower frequencies, respectively. (D) Heat maps of power spectrograms, with base frequencies marked by asterisks. (E) Pooled responses of $n=3 \mathrm{iCMC}$ cultures to $10 \mu \mathrm{mol} / \mathrm{L}$ doxorubicin (red: $2 \mathrm{~h}$ treatment, blue: $5 \mathrm{~h}$ treatment) and to a subsequent recovery period (green). ${ }^{\#} P<0.05$. (F) Average waveform of the beat pattern does not indicate a substantially altered raise or decay time of iCMC contractility. Scale bars $100 \mu \mathrm{m}$.

$\mathrm{K}$ ions hence lengthen the plateau and repolarization phases. The net impact on the beat frequency of these opposing effects was sensitive to model parameters. The default parameters of Model B, however, predicted an increase in beat frequency (Figure 7D).

In addition to ion channel modulators, we demonstrate the sensitivity of the optical flow/PIV method by determining the well-known cardiotoxic side effects of a chemotherapeutic agent, doxorubicin ${ }^{[29]}$. For this, the iCMCs were cultured in the presence and absence of $10 \mu \mathrm{mol} / \mathrm{L}$ doxorubicin for $5 \mathrm{~h}$. Doxorubicin exposure resulted in a transient increase, then an irreversible decrease of the $\mathrm{SBF}$ to $\mathrm{NBF}=0.60 \pm 0.05(P<0.01$, Figure 8). Beat patterns indicate that the decreased SBF is mainly due to the lengthening of the resting phase: the contraction and relaxation phases appear to be unchanged. The SBA was also irreversibly reduced by doxorubicin to $\mathrm{NBA}=0.2 \pm 0.05$ $(P<0.01)$. These data clearly suggest that our PIV method is sensitive enough to detect clinically relevant cardiotoxicity.

\section{Discussion}

Currently, the incidence of cardiac and non-cardiac drug reac- tions is excessive in patients due to the limited capacity of preclinical screening to detect cardiotoxicity. Development of new medications that are free from adverse cardiac side effects is especially hampered by the lack of an appropriate in vitro assay for human cardiac tissue.

Human iCMCs emerge as an inexpensive and easily available source of human heart cells for pharmacological screening and drug toxicity assays. In particular, a recent study has shown that iCMCs derived from a long QT syndrome (LQTS) patient can be used to evaluate the potency of existing and novel pharmacological agents that are regulating various ion channels ${ }^{[9]}$. Similarly, iCMCs derived from dilated cardiomyopathy (DCM) patients resemble to some extent to the morphological and functional phenotypes of DCM and are serving as a useful tool for exploring disease mechanism and drug screening $^{[11]}$. This method also circumvents many hurdles associated with transgenic animal models that have been previously used for studying several cardiovascular disorders. In particular, iCMCs with a certain genetic makeup can be obtained much faster than the corresponding transgenic mice. Furthermore, human and mouse cells have physiological dif- 
ferences, like their intrinsic heartbeat frequency, that can also modulate the chronotropic effect of certain pharmacological compounds. Usage of iCMCs also reduces reliance on animal models and may indicate problems before the expensive clinical trial phases of the approval process. Current in vitro safety pharmacology guidelines suggest that all new drugs need to undergo evaluation for major functional changes in cardiac ion channels to rule out potential cardiotoxicity. While iCMCs are often immature when compared with primary adult cardiomyocytes, the iCMCs generated in this study were mature and phenotypically and morphologically closely related to adult CMCs as confirmed by both CMC-specific protein expression and electron microscopic image analyses.

Although there are a few drug screening models available utilizing ESC or iPSC-derived cardiomyocytes, most published approach uses either $\mathrm{Ca}^{2+}$ transient imaging or patch clamping, which restricts their application in high-throughput settings ${ }^{[4]}$. In this study, we systematically analyzed small molecule mediated spatio-temporal functional effects and cardiotoxicity in iCMCs using our previously described contactfree optical flow/PIV method ${ }^{[5]}$. Changes in the frequency and amplitude of the spontaneous CMC beating activity were characterized by an unbiased Fourier spectrogram analysis that represents frequency shifts in a heat map format. Fourier spectrogram heat maps also allow to determine pharmacokinetic parameters such as the time required to reach peak effect, or the stability of the compound in this model system. Our approach is simple, fast and requires only unsophisticated phase contrast video microscopic imaging capability to assess how drugs affect the kinetics of spontaneous beating activity of cardiomyocytes. Here we analyzed various modulators of $\mathrm{Ca}^{2+}, \mathrm{K}^{+}$and $\mathrm{Na}^{+}$ion channel dynamics, key regulators of action potential generation.

Studies have reported that L-type calcium channels play critical roles in cardiac contractile function ${ }^{[30]}$. Isoproterenol is a potent nonselective $\beta$-adrenergic agonist which activates the cAMP pathway and enhances contractile function. However, increased signaling also leads to cardiac hypertrophy and in some cases to heart failure ${ }^{[31]}$. Clinically, isoproterenol is used to treat bradycardia, congestive heart failure and heart block $^{[32]}$. To evaluate the effect of this drug in our in vitro system, we have analyzed the spontaneous beating activity of CMCs treated with isoproterenol using our optical flow/ PIV method. We observed significantly increased SBF and an unaffected SBA in the presence of isoproterenol. These data suggest that in agreement with the available clinical findings, isoproterenol has a positive chronotropic effect on CMCs.

NS1643, an agonist of rapidly activating $\mathrm{K}^{+}$channels $\left(I_{\mathrm{Kr}}\right)$, is an antiarrhythmic drug increasing heartbeat frequency and contractile force $^{[33,34]}$. Most commonly, QT interval prolongation is due to reductions in either the rapidly activating $\left(I_{\mathrm{Kr}}\right)$ or by slowly activating $\left(I_{\mathrm{Ks}}\right)$ mediated delayed repolarizing cardiac $\mathrm{K}^{+}$current. Clinically, NS1643 has been shown to affect hERG channels, shorten the QT interval and increase the beat amplitude. Overall, our data demonstrate that day 14 iCMCs were responsive to NS1643 by increasing SBF and SBA and were in agreement with the clinical benefits. These effects were reversed by the potassium channel blocker, E4031, which is used to prolong repolarization and increase maximum diastolic potential. E4031 is an experimental class III antiarrhythmic drug that blocks $\mathrm{K}^{+}$channels of hERG type ${ }^{[35]}$. In a recent study, E4031 was used to suppress the spontaneous development of different beating frequencies among cardiomyocytes $^{[36]}$. In our in vitro study, we observed that E4031 mediated reduction of the $I_{\mathrm{Kr}}$ current prolonged the contraction of $\mathrm{CMCs}$, likely by increasing the duration of the repolarization phase of the cardiac action potential. This effect also leads to a decreased SBF. Thus, in agreement with its clinical purpose, E4031 exerts a negative chronotropic and ionotropic effects on iCMCs. In general, our analysis supports the concept of hERG channel modulators as novel antiarrhythmic agents.

Veratridine is a steroid-derived alkaloid from plant product of Liliaceae family. It functions by preferentially binding to activated $\mathrm{Na}^{+}$channels causing their persistent activation ${ }^{[37]}$. Recent studies have shown that veratridine also affects the function of $\mathrm{Na}^{+}$channels in $\mathrm{CMCs}^{[38,39]}$. Our in vitro data also indicates a negative chronotropic effect of veratridine on iCMCs in agreement with an earlier report ${ }^{[39]}$. In contrast, mexiletine is a non-selective voltage gated $\mathrm{Na}^{+}$channel blocker, which belongs to the class $1 \mathrm{~B}$ of anti-arrhythmic group of medicine. Clinically it is used to reduce ventricular tachycardia and to stabilize the heart rhythm in cases like ventricular premature beat. It has also been shown that mexiletine reduces $\mathrm{Na}^{+}$current in atrial and ventricular myocytes ${ }^{[40]}$. In this study, the SBF of iCMCs was significantly lowered by mexiletine without changing the SBA. Again, these findings are in agreement with earlier clinical findings ${ }^{[40]}$.

The drugs we have used in this study are well defined cardiac modulators and exert relatively strong effects on cardiac functions. To mimic cardiotoxicity screening of an anti-cancer drug, we also analyzed iCMCs before and during doxorubicin treatment. Our data clearly shows that there is an irreversible reduction in both beat frequency and amplitude which is in agreement with an earlier report ${ }^{[29]}$.

In conclusion, we demonstrated the feasibility of measuring clinically relevant CMC functions through a non-contact optical analysis yielding both spontaneous beat frequency and amplitude. Comparing with the available methods, our new method has several advantages such as being manipulationfree, less invasive, user friendly, inexpensive and has a superior efficiency to evaluate the adverse effect of drugs including anti-cancer drugs. Thus, we expect our CMC monitoring method to become a useful and novel tool to assess drug effects and cardiotoxicity in preclinical drug screening.

\section{Acknowledgements}

This work was supported by the NIH (GM102801 to Andras CZIROK), the American Heart Association (16GRNT31030030 to Andras CZIROK and 16GRNT30950010 to Johnson RAJASINGH), National Institutes of Health COBRE grant P20GM104936 (to Johnson RAJASINGH). 


\section{Author contribution}

Andras CZIROK, Sheeja RAJASINGH, and Johnson RAJASINGH conceived the experiments; Sheeja RAJASINGH, Saheli SAMANTA and Zhi-gang ZHOU conducted the experiments; Andras CZIROK and Dona Greta ISAI performed the computational aspects of the research; Andras CZIROK, Johnson RAJASINGH, Buddhadeb DAWN, and Dona Greta ISAI analyzed the results. All authors reviewed the manuscript.

\section{Supplementary information}

Supplementary information is available at the website of Acta Pharmacologica Sinica.

\section{References}

1 Siramshetty VB, Nickel J, Omieczynski C, Gohlke BO, Drwal MN, Preissner R. WITHDRAWN-a resource for withdrawn and discontinued drugs. Nucleic Acids Res 2016; 44: D1080-6.

2 Lee EK, Kurokawa YK, Tu R, George SC, Khine M. Machine learning plus optical flow: a simple and sensitive method to detect cardioactive drugs. Sci Rep 2015; 5: 11817.

3 Itzhaki I, Maizels L, Huber I, Gepstein A, Arbel G, Caspi O, et al. Modeling of catecholaminergic polymorphic ventricular tachycardia with patient-specific human-induced pluripotent stem cells. J Am Coll Cardiol 2012; 60: 990-1000.

4 Doherty KR, Talbert DR, Trusk PB, Moran DM, Shell SA, Bacus S. Structural and functional screening in human induced-pluripotent stem cell-derived cardiomyocytes accurately identifies cardiotoxicity of multiple drug types. Toxicol Appl Pharmacol 2015; 285: 51-60.

5 Rajasingh S, Thangavel J, Czirok A, Samanta S, Roby KF, Dawn B, et al. Generation of functional cardiomyocytes from efficiently generated human iPSCs and a novel method of measuring contractility. PLoS One 2015; 10: e0134093.

6 Burridge PW, Keller G, Gold JD, Wu JC. Production of de novo cardiomyocytes: human pluripotent stem cell differentiation and direct reprogramming. Cell Stem Cell 2012; 10: 16-28.

7 Burridge PW, Matsa E, Shukla P, Lin ZC, Churko JM, Ebert AD, et al. Chemically defined generation of human cardiomyocytes. Nat Methods 2014; 11: 855-60.

8 Karakikes I, Termglinchan V, Wu JC. Human-induced pluripotent stem cell models of inherited cardiomyopathies. Curr Opin Cardiol 2014; 29: 214-9.

9 Itzhaki I, Maizels L, Huber I, Zwi-Dantsis L, Caspi O, Winterstern A, et al. Modelling the long QT syndrome with induced pluripotent stem cells. Nature 2011; 471: 225-9.

10 Narsinh K, Narsinh KH, Wu JC. Derivation of human induced pluripotent stem cells for cardiovascular disease modeling. Circ Res 2011; 108: 1146-56.

11 Sun N, Yazawa M, Liu J, Han L, Sanchez-Freire V, Abilez OJ, et al. Patient-specific induced pluripotent stem cells as a model for familial dilated cardiomyopathy. Sci TransI Med 2012; 4: 130ra47.

12 Mandel Y, Weissman A, Schick R, Barad L, Novak A, Meiry G, et al. Human embryonic and induced pluripotent stem cell-derived cardiomyocytes exhibit beat rate variability and power-law behavior. Circulation 2012; 125: 883-93.

13 Malan D, Zhang M, Stallmeyer B, Muller J, Fleischmann BK, SchulzeBahr E, et al. Human iPS cell model of type 3 long QT syndrome recapitulates drug-based phenotype correction. Basic Res Cardiol 2016; 111: 14

14 Seki T, Yuasa S, Kusumoto D, Kunitomi A, Saito Y, Tohyama S, et al.
Generation and characterization of functional cardiomyocytes derived from human T cell-derived induced pluripotent stem cells. PLoS One 2014; 9: e85645.

15 Thangavel J, Malik AB, Elias HK, Rajasingh S, Simpson AD, Sundivakkam PK, et al. Combinatorial therapy with acetylation and methylation modifiers attenuates lung vascular hyperpermeability in endotoxemia-induced mouse inflammatory lung injury. Am J Pathol 2014; 184: 2237-49.

16 Rajasingh J, Lambers E, Hamada H, Bord E, Thorne T, Goukassian I, et al. Cell-free embryonic stem cell extract-mediated derivation of multipotent stem cells from NIH3T3 fibroblasts for functional and anatomical ischemic tissue repair. Circ Res 2008; 102: e107-17.

17 Rajasingh J, Thangavel J, Siddiqui MR, Gomes I, Gao XP, Kishore R, et al. Improvement of cardiac function in mouse myocardial infarction after transplantation of epigenetically-modified bone marrow progenitor cells. PLoS One 2011; 6: e22550.

18 Czirok A, Isai DG, Kosa E, Rajasingh S, Kinsey W, Neufeld Z, et al. Optical-flow based non-invasive analysis of cardiomyocyte contractility. Sci Rep 2017; 7: 10404.

19 Aleksandrova A, Czirok A, Szabo A, Filla MB, Hossain MJ, Whelan $\mathrm{PF}$, et al. Convective tissue movements play a major role in avian endocardial morphogenesis. Dev Biol 2012; 363: 348-61.

20 Zamir EA, Czirok A, Cui C, Little CD, Rongish BJ. Mesodermal cell displacements during avian gastrulation are due to both individual cell-autonomous and convective tissue movements. Proc Natl Acad Sci U S A 2006; 103: 19806-11.

21 McAllister RE, Noble D, Tsien RW. Reconstruction of the electrical activity of cardiac Purkinje fibres. J Physiol 1975; 251: 1-59.

22 Guo L, Abrams RM, Babiarz JE, Cohen JD, Kameoka S, Sanders MJ, et al. Estimating the risk of drug-induced proarrhythmia using human induced pluripotent stem cell-derived cardiomyocytes. Toxicol Sci 2011; 123: 281-9.

23 Li X, Zhang R, Zhao B, Lossin C, Cao Z. Cardiotoxicity screening: a review of rapid-throughput in vitro approaches. Arch Toxicol 2016; 90 : 1803-16.

24 Bilet A, Bauer CK. Effects of the small molecule HERG activator NS1643 on Kv11.3 channels. PLoS One 2012; 7: e50886.

25 Hara M, Danilo P Jr, Rosen MR. Effects of gonadal steroids on ventricular repolarization and on the response to E4031. J Pharmacol Exp Ther 1998; 285: 1068-72.

26 Altomare C, Pianezzi E, Cervio E, Bolis S, Biemmi V, Benzoni P, et al. Human-induced pluripotent stem cell-derived cardiomyocytes from cardiac progenitor cells: effects of selective ion channel blockade. Europace 2016; 18: iv67-iv76.

27 Chevalier M, Amuzescu B, Gawali V, Todt H, Knott T, Scheel O, et al. Late cardiac sodium current can be assessed using automated patchclamp. F1000Research 2014; 3: 245.

28 Clements M, Millar V, Williams AS, Kalinka S. Bridging functional and structural cardiotoxicity assays using human embryonic stem cellderived cardiomyocytes for a more comprehensive risk assessment. Toxicol Sci 2015; 148: 241-60.

29 Maillet A, Tan K, Chai X, Sadananda SN, Mehta A, Ooi J, et al. Modeling doxorubicin-induced cardiotoxicity in human pluripotent stem cell derived-cardiomyocytes. Sci Rep 2016; 6: 25333.

30 Trautwein W, Hescheler J. Regulation of cardiac L-type calcium current by phosphorylation and G proteins. Annu Rev Physiol 1990; 52: $257-74$

31 Copik AJ, Baldys A, Nguyen K, Sahdeo S, Ho H, Kosaka A, et al. Isoproterenol acts as a biased agonist of the alpha-1A-adrenoceptor that selectively activates the MAPK/ERK pathway. PLoS One 2015; 10: e0115701. 
32 Gravning J, Ahmed MS, Qvigstad E, Krobert K, Edvardsen T, Moe IT, et al. Connective tissue growth factor/CCN2 attenuates beta-adrenergic receptor responsiveness and cardiotoxicity by induction of $\mathrm{G}$ proteincoupled receptor kinase-5 in cardiomyocytes. Mol Pharmacol 2013; 84: 372-83.

33 Li P, Chen X, Zhang Q, Zheng Y, Jiang $H$, Yang $H$, et al. The human ethera-go-go-related gene activator NS1643 enhances epilepsy-associated KCNQ channels. J Pharmacol Exp Ther 2014; 351: 596-604.

34 Hansen RS, Diness TG, Christ T, Demnitz J, Ravens U, Olesen SP, et al. Activation of human ether-a-go-go-related gene potassium channels by the diphenylurea 1,3-bis-(2-hydroxy-5-trifluoromethyl-phenyl)-urea (NS1643). Mol Pharmacol 2006; 69: 266-77.

35 Shryock JC, Song Y, Rajamani S, Antzelevitch C, Belardinelli L. The arrhythmogenic consequences of increasing late $I_{\mathrm{Na}}$ in the cardiomyocyte. Cardiovasc Res 2013; 99: 600-11.

36 Eng G, Lee BW, Protas L, Gagliardi M, Brown K, Kass RS, et al.
Autonomous beating rate adaptation in human stem cell-derived cardiomyocytes. Nat Commun 2016; 7: 10312.

37 Cao Z, Gerwick WH, Murray TF. Antillatoxin is a sodium channel activator that displays unique efficacy in heterologously expressed rNav1.2, rNav1.4 and rNav1.5 alpha subunits. BMC Neurosci 2010; 11: 154.

38 Li X, Shen L, Zhao F, Zou X, He Y, Zhang F, et al. Modification of distinct ion channels differentially modulates $\mathrm{Ca}^{2+}$ dynamics in primary cultured rat ventricular cardiomyocytes. Sci Rep 2017; 7: 40952.

39 Frotscher R, Koch JP, Staat M. Computational investigation of drug action on human-induced stem cell-derived cardiomyocytes. J Biomech Engineer 2015, 137: 071002-071002-7

40 Suzuki T, Morishima M, Kato S, Ueda N, Honjo H, Kamiya K. Atrial selectivity in $\mathrm{Na}^{+}$channel blockade by acute amiodarone. Cardiovasc Res 2013; 98: 136-44. 